\title{
A Sublaminate Generalized Unified Formulation for the analysis of composite structures
}

\author{
Michele D'Ottavio
}

\section{Introduction}

The ongoing transition of composite structures from secondary structural applications to primary load-bearing structures demands dedicated models that are capable of an accurate determination of both gross and local response. The great interest in theoretical and computational models for multilayered panels is witnessed by the large number of reviews appraising the developments in this field, e.g., [1-6]. The very large majority of models has been constructed following the axiomatic approach, in which some constraining hypotheses are postulated about the behavior along the panel's thickness of the displacement field (displacement approach), the stress field (stress approach) or both displacement and stress fields (mixed approach) [5]. The present contribution also focuses on this axiomatic approach.

A convenient classification of axiomatic models for composite multilayered panels identifies Equivalent Single Layer (ESL) and Layer-Wise (LW) models [7]. In an ESL approach, the heterogeneous layup is homogenized to represent an anisotropic material that includes membrane-bending as well as extension-shear coupling. The resulting computational models have a number of unknowns that is independent of the number of plies constituting the panel. In a LW approach, the constraining hypotheses are formulated in each layer the composite layup has been subdivided

E-mail address: michele.d_ottavio@u-paris10.fr into. This results in an overall number of unknowns that depends on the number of layers. In-between ESL and LW theories, Zig-Zag theories allow to represent to a certain extent the characteristic heterogeneity of composite structures while still retaining a number of unknowns that is independent of the number of plies constituting the multilayered panel [8].

A direct implementation of the LW approach consists in assuming the same behavior for all physical plies constituting the multilayered structure. This model has the evident drawback of involving a very large number of unknowns when the composite structure is made of a large number of plies. The total number of unknowns of a LW model may be optimized in two manners. On the one hand, the number of layers can be minimized, i.e., several physical plies can be regrouped into one sublaminate whenever it appears convenient. On the other hand, the number of unknown parameters of the models of each sublaminate may be minimized by introducing dedicated assumptions that account for its geometrical and/or physical properties. This is a natural approach for sandwich panels, in which different hypotheses are formulated for the thin and stiff faces and the relatively thick and soft core, see, e.g. $[9,4]$. Reddy's LW model $[10,11]$ implements these aspects by subdividing the layup into an arbitrary number of sublaminates and giving the possibility of defining the through-thickness behavior of the displacement field in each sublaminate independently.

A very flexible modeling tool for composite structures has been introduced by Carrera $[12,13]$ in terms of a Unified Formulation, 


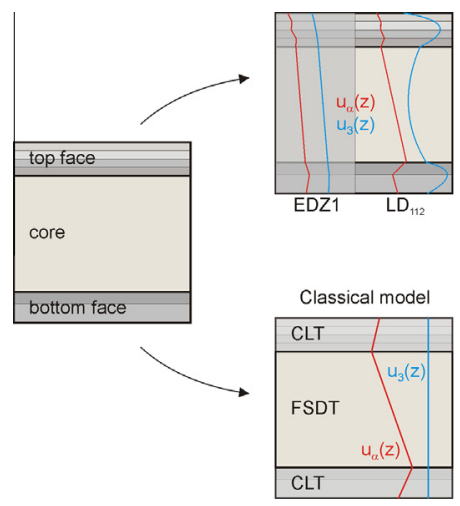

\section{CUFIGUF models:}

Whole layup described either ESL or LW

Same approximation order $N$ used for all plies

Fig. 1. The proposed Sublaminate-GUF (S-GUF) broadens the flexibility of CUF/GUF by allowing to formulate different GUF-type models for separate sublaminates.

i.e., a compact index notation that permits to write the governing equations of different models within a unique computer program. The advantages of such an approach are multiple: it is possible to directly assess with the same computer code several models for establishing their range of applicability [14-16]; to determine the optimal model for a given problem in terms of accuracy versus computational cost [17,18]; or to implement a global-local submodeling approach by coupling less accurate models with low computational cost with more accurate and computationally expensive models $[19,20]$.

In the original Carrera's Unified Formulation (CUF), the constraining hypotheses for the displacement field can be introduced within the weak forms expressed by the Principle of Virtual Displacements (PVD) or Reissner's Mixed Variational Theorem (RMVT) [21]. RMVT allows to additionally introduce independent approximations for the transverse stress field, for instance for a priori fulfilling the interlayer equilibrium. In CUF, the displacement field can be described in either LW or ESL manner, possibly including a Zig-Zag function for improving the ESL description, whereas transverse stresses are always taken LW. Moreover, the order of the polynomial approximation through the thickness of the panel (in an ESL description) or of the layer (in a LW description) may be freely selected, but it is taken to be the same for all unknown functions. This latter limitation has been removed by the Generalized Unified Formulation (GUF) proposed by Demasi [22,23], where different orders can be introduced for the approximation of different field variables. Demasi [24] further extended his PVD-based GUF upon allowing independent ESL or LW descriptions for each displacement component. However, in both GUF and CUF the same hypotheses (i.e., ESL or LW description and approximation order) are used for all the plies of the composite. A recent development dedicated to sandwich plates extended the RMVT-based CUF to use an ESL description of the displacement field at the composite face sheets level, while using a Layer-Wise description for the sandwich level [25]. Similar ideas have been also recently implemented by Messina [26] by referring to Adaptive-Global Piecewise-Smooth Functions (A-GPSF).

The present contribution extends in a systematic way the above Unified Formulations by introducing the sublaminate concept of Reddy [10]: different hypotheses expressed through the most flexible GUF are formulated at a sublaminate level. As a result, each field variable pertaining to the PVD or RMVT approach, can be described in either ESL or LW manner and with an independent order of the polynomial approximation inside each arbitrarily defined sublaminate. The computational cost can be hence optimized by enriching the approximation - within the variable kinematics modeling freedom offered by the Unified Formulation - only in those sublaminates that deserve a refined description. This is the main novelty of the present work and is schematically illustrated in Fig. 1. It appears useful to emphasize that the proposed formulation is an extension of existing variable kinematics modeling approaches by comparing it with the latest developments concerning GUF and CUF reported in [24,25], respectively. On the one hand, the PVD-based development of [24] is extended by enhancing the modeling flexibility through the introduction of sublaminates and by addressing also RMVT-based models; note that all established GUF models can be retrieved as special cases of the proposed formulation by representing the whole composite section as only one sublaminate. On the other hand, the approach proposed in [25] is extended by expressing the assumptions within GUF instead of CUF and by allowing also the use of PVD-models. Therefore, the proposed S-GUF permits a further optimization of the computational cost, with respect to [24] by allowing to select different approximations for different sublaminates and with respect to [25] by allowing to use different approximations for different field variables in each sublaminate.

The paper is organized as follows. A literature review is proposed in Section 2 for introducing several models that will be used in the numerical evaluations proposed in the paper. Section 3 details out the Sublaminate-GUF. Section 4 proposes a naming convention for the models formulated in the framework of S-GUF. The numerical assessment in Section 5 addresses bending problems of simply supported, rectangular and orthotropic sandwich plates for which a closed-form Navier-type solution exists. Section 6 closes the paper with a brief summary and an outlook towards further work.

\section{Literature review}

Typical examples of ESL models are the Classical Laminate Theory (CLT), and the First-order Shear Deformation Theory (FSDT) [11]. Both CLT and FSDT rely on the plane stress hypothesis discarding the transverse normal stress and refer to the reduced 2D constitutive law [27]. The accuracy of FSDT is known to depend on problem-specific Shear Correction Factors (SCF), which numerically tune the transverse shear deformability of the model [28].

Higher-order Shear Deformation Theories (HSDT) are constructed by introducing more permissive hypotheses about the transverse shear deformation, although the plane stress condition is still retained. Shear Correction Factors are no longer required in HSDT. Ambartsumyan's and Reddy's third-order theory $[29,30]$, based on a mixed approach with a quadratic variation of the transverse shear stresses and a cubic one for the in-plane displacements, or the displacement-based Sinus model of Touratier [31] count among the most representative HSDT. The discard of the transverse normal stress appears justified in absence of significant loading gradients occurring over a length proportional to the panel thickness [32]. The seminal work of 
Lo et al. [33] enhances the third-order theory by including a quadratic variation of the transverse deflection and, hence, a linear transverse stretch. The full $3 \mathrm{D}$ constitutive law is thus retained. It is worth noticing that the linear transverse stretch prevents the so-called Poisson locking [34]. High-order ESL models may give useful estimates of the global response, e.g., fundamental frequencies [6].

A noticeable enhancement of ESL models can be achieved by referring to Zig-Zag theories, in which a piecewise linear displacement field is superimposed to a classical ESL description. The resulting displacement field has discontinuous slopes at layer interfaces (Zig-Zag shape) and the consequent discontinuous transverse strains allow to fulfill (at least to a certain extent) the interlaminar equilibrium between plies with different material properties [35]. The importance of the Zig-Zag term depends on the mismatch of the mechanical properties of adjacent plies, which is particularly strong, e.g., between skins and core of a sandwich structure [36-38]. A simple and material-independent Zig-Zag function could be conveniently introduced within the mixed approach expressed by RMVT [39-41]. This so-called Murakami's Zig-Zag Function (MZZF) has been shown to be able to substantially enhance the behavior of displacement-based models as well $[42,43]$.

The survey by Carrera and Brischetto [44] has shown that the errors in the response predicted by ESL models, even those including the Zig-Zag effect, increase with the panel thickness and the strength of the material properties' mismatch. In these cases, a LW approach appears thus necessary. Several works report LW models in which the same kinematic model is used for both faces and core of sandwich panels. For instance, Librescu and Hause [45] employ FSDT, Pai and Palazotto [46] apply the refined model of Lo et al. [33] and D'Ottavio and Polit [47] use the most accurate RMVT-based CUF model LM4.

However, when modeling sandwich structures it is customary to employ different approximations for the faces and the core [9]. Low-order models are usually chosen for the thin faces, such as pure membrane [48], CLT [49-52] or FSDT [53]. A much larger variety of models has been used to represent the thick and soft core. Honeycomb cores are often considered to work in pure transverse shear [49] or in anti-plane stress (zero membrane stiffness) [50]. Several different assumptions have been introduced for modeling the core as a 3D elastic continuum: for instance, the transverse shear strain has been taken to vary linearly [51] or quadratically [52] across the core thickness; and the transverse normal stress has been assumed to be constant [53] or linear [52]. Most of the plate models discussed insofar can be obtained as special cases of the Sublaminate-GUF presented in the following.

\section{The Sublaminate-Generalized Unified Formulation}

\subsection{Geometric description of the composite plate}

We consider a plate that occupies a volume $V=\Omega \times\left[-\frac{H}{2}, \frac{H}{2}\right]$, where $\Omega=[0, a] \times[0, b]$ is the reference surface in the $(x, y)=\left(x_{1}, x_{2}\right)$ plane and $H$ is the plate thickness along the transverse direction $x_{3}=z$. As illustrated in Fig. 2 (left), the plate is composed of $p=1,2 \ldots N_{p}$ homogeneous, orthotropic and perfectly bonded plies of thickness $h_{p}$, which is assumed constant over the $x y$-plane. Fig. 2 (right) shows how the composite cross-section is here subdivided into $k=1,2 \ldots N_{l}$ numerical layers, the thickness of the $k$ th layer being denoted $h_{k}$. Throughout the work, the subscript $k$ is the layer index while the subscript $p$ the ply index.

Numerical layers could be employed in the framework of conventional CUF and GUF to subdivide physical plies, i.e. $h_{k} \leqslant h_{p}$.

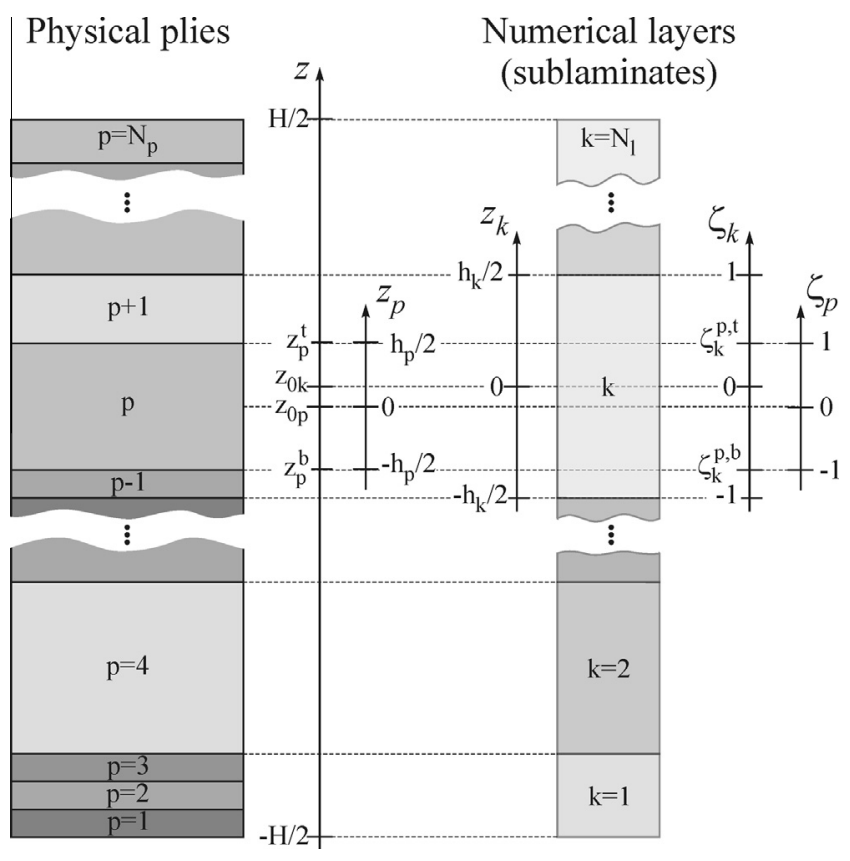

Fig. 2. Multilayered plate as an assembly of $N_{p}$ physical plies (left) and $N_{l}$ numerical layers (right). Global $z$, layer-specific $z_{k}$ and ply-specific $z_{p}$ coordinates are used for the description of the model along with the non-dimensional layer- and ply-specific coordinates $\zeta_{k}$ and $\zeta_{p}$.

The present approach extends the notion of "numerical layer" by introducing the possibility of regrouping several physical plies into one numerical layer, which leads to the definition of sublaminates. Let the generic $k$ th sublaminate be composed of $N_{p}^{k}$ physical plies, ranging from the ply $p=p_{1}^{k}$ located at its bottom (first ply of the sublaminate) to the ply $p=p_{N}^{k}=p_{1}^{k}+N_{p}^{k}-1$ located at its top (last ply of the sublaminate). So, the case $N_{p}^{k}=1$ can refer to the conventional definition of numerical layer $\left(h_{k} \leqslant h_{p}\right)$, whereas the case $N_{p}^{k}>1$ with $h_{k}>h_{p}$ refers to the new possibility of defining sublaminates inside the composite plate. Throughout the text, "numerical layer" and "sublaminate" shall hence be interchangeably called "layer" without loss of clarity.

Several thickness coordinates are introduced for the description of the composite cross-section, see Fig. 2. In the global coordinate $z \in\left[-\frac{H}{2}, \frac{H}{2}\right]$, the positions of each ply $p$ and of each layer $k$ are identified by their mid-plane coordinates, denoted $z_{0 p}$ and $z_{0 k}$, respectively, which are obtained from the coordinates of the corresponding top and bottom interfaces:

$z_{0 k}=\frac{z_{k}^{t}+z_{k}^{b}}{2}$ and $z_{0 p}=\frac{z_{p}^{t}+z_{p}^{b}}{2}$

Layer-specific as well as ply-specific coordinates are introduced as

$z_{k}=z-z_{0 k}, z_{k} \in\left[-\frac{h_{k}}{2}, \frac{h_{k}}{2}\right]$ and $z_{p}=z-z_{0 p}, z_{p} \in\left[-\frac{h_{p}}{2}, \frac{h_{p}}{2}\right]$

Non-dimensional coordinates are further introduced as

$\zeta_{k}=\frac{2 z_{k}}{h_{k}}, \zeta_{k} \in[-1,1]$ and $\zeta_{p}=\frac{2 z_{p}}{h_{p}}, \zeta_{p} \in[-1,1]$

From Eqs. (2) and (3) one obtains the non-dimensional coordinates $\zeta_{k}^{(p, t)}$ and $\zeta_{k}^{(p, b)}$ of the top and bottom interfaces of the physical ply $p$ inside the $k$ th sublaminate: 
$\zeta_{k}^{(p, t)}=\zeta_{k}\left(z_{p}^{t}\right)=\frac{2}{h_{k}}\left(z_{p}^{t}-z_{0 k}\right) \quad$ and $\quad \zeta_{k}^{(p, b)}=\zeta_{k}\left(z_{p}^{b}\right)=\frac{2}{h_{k}}\left(z_{p}^{b}-z_{0 k}\right)$

(see Fig. 2). Finally, the relation between the non-dimensional plyspecific and the layer-specific coordinates is obtained as

$\left.\zeta_{p}=\frac{h_{k}}{h_{p}} \zeta_{k}+\frac{2}{h_{p}}\left(z_{0 k}-z_{0 p}\right)=\frac{2}{\zeta_{k}^{(p, t)}-\zeta_{k}^{(p, b)}} \zeta_{k}-\frac{\zeta_{k}^{(p, t)}+\zeta_{k}^{(p, b)}}{2}\right)$

which verifies that $\zeta_{p}\left(\zeta_{k}=\zeta_{k}^{(p, t)}\right)=1$ and $\zeta_{p}\left(\zeta_{k}=\zeta_{k}^{(p, b)}\right)=-1$.

\subsection{Variational statements}

The constraining hypotheses defining the plate model in axiomatic sense are introduced by referring to variational statements. The proposed S-GUF models are constructed following the displacement-based approach expressed by the PVD or Reissner's mixed approach expressed by RMVT.

\subsubsection{Displacement-based approach}

The PVD expresses the weak form of the equilibrium equations in terms of admissible, virtual variations of displacements $u_{i}(x, y, z)$ as

$\iint_{\Omega}\left\{\int_{H} \delta \epsilon_{\alpha \beta}^{(G)} \sigma_{\alpha \beta}^{(H)}+\delta \epsilon_{i 3}^{(G)} \sigma_{i 3}^{(H)} \mathrm{d} z\right\} \mathrm{d} x \mathrm{~d} y=\iint_{\Omega} \delta u_{i} t_{i} \mathrm{~d} x \mathrm{~d} y$

where Einstein's summation convention has been used with Greek indexes $\alpha, \beta \in\{1,2\}$ and Latin index $i \in\{1,2,3\}$. The imposed tractions (external forces per unit surface) are denoted by $t_{i}$. The inplane and transverse strains are defined by the geometric relations (superscript $(G)$ )

$\epsilon_{\alpha \beta}^{(G)}=\frac{1}{2}\left(u_{\alpha, \beta}+u_{\beta, \alpha}\right) ; \epsilon_{i 3}^{(G)}=\frac{1}{2}\left(u_{i, 3}+u_{3, i}\right)$

where the compact notation $u_{, i}=\partial u / \partial x_{i}$ is used. The generalized Hooke's law (superscript $(H)$ ) defines the corresponding in-plane and transverse stresses in each ply $p$ (superscript $(p)$ ):

$\left[\begin{array}{c}\sigma_{\alpha \beta}^{(H)} \\ \sigma_{i 3}^{(H)}\end{array}\right]^{(p)}=\left[\begin{array}{ll}\widetilde{C}_{\alpha \beta \gamma \delta} & \widetilde{C}_{\alpha \beta j 3} \\ \widetilde{C}_{i 3 \gamma \delta} & \widetilde{C}_{i 3 j 3}\end{array}\right]^{(p)}\left[\begin{array}{c}\epsilon_{\gamma \delta}^{(G)} \\ \epsilon_{j 3}^{(G)}\end{array}\right]$

Introducing Voigt notation and regrouping the in-plane and transverse components of the stress and strain vectors as

$\sigma_{\omega}=\left[\sigma_{1} \sigma_{2} \sigma_{6}\right]^{\top} ; \quad \epsilon_{\omega}=\left[\epsilon_{1} \epsilon_{2} \epsilon_{6}\right]^{\top} ; \quad \sigma_{n}=\left[\sigma_{4} \sigma_{5} \sigma_{3}\right]^{\top} ;$

$\epsilon_{n}=\left[\epsilon_{4} \epsilon_{5} \epsilon_{3}\right]^{\top}$

Hooke's law Eq. (8) can be written as

$$
\left[\begin{array}{c}
\sigma_{\omega}^{(H)} \\
\sigma_{n}^{(H)}
\end{array}\right]^{(p)}=\left[\begin{array}{ll}
\widetilde{C}_{\omega \omega} & \widetilde{C}_{\omega n} \\
\widetilde{C}_{\omega n}^{\top} & \widetilde{C}_{n n}^{(p)}
\end{array}\right]^{(G)}\left[\begin{array}{l}
\epsilon_{\omega}^{(G)} \\
\epsilon_{n}^{(G)}
\end{array}\right]
$$

where the superscript ${ }^{\top}$ indicates transposition. The internal virtual work expressed by the PVD Eq. (6) can then be written as

$$
\begin{aligned}
\delta W_{\text {int }}= & \iint_{\Omega}\left\{\int_{H} \delta \epsilon_{\omega}^{(G)} \sigma_{\omega}^{(H)}+\delta \epsilon_{n}^{(G) \top} \sigma_{n}^{(H)} \mathrm{d} z\right\} \mathrm{d} x \mathrm{~d} y \\
= & \iint_{\Omega}\left\{\int_{H} \delta \epsilon_{\omega}^{(G)}{ }^{\top} \widetilde{C}_{\omega \omega}^{(p)} \epsilon_{\omega}^{(G)}+\delta \epsilon_{\omega}^{(G)} \widetilde{C}_{\omega n}^{(p)} \epsilon_{n}^{(G)}+\delta \epsilon_{n}^{(G) \top} \widetilde{C}_{\omega n^{(p)}}^{\top} \epsilon_{\omega}^{(G)}\right. \\
& \left.+\delta \epsilon_{n}^{(G) \top} \widetilde{C}_{n n}^{(p)} \epsilon_{n}^{(G)} \mathrm{d} z\right\} \mathrm{d} x \mathrm{~d} y
\end{aligned}
$$

\subsubsection{Reissner's mixed approach}

In the case of RMVT, the virtual internal work is expressed in terms of independent variations of the admissible displacements $u_{i}(x, y, z)$ and of the transverse stresses $\sigma_{i 3}(x, y, z)[21]$ :

$$
\begin{aligned}
& \iint_{\Omega}\left\{\int_{H} \delta \epsilon_{\alpha \beta}^{(G)} \sigma_{\alpha \beta}^{(C)}+\delta \epsilon_{i 3}^{(G)} \sigma_{i 3}+\delta \sigma_{i 3}\left(\epsilon_{i 3}^{(G)}-\epsilon_{i 3}^{(C)}\right) \mathrm{d} z\right\} \mathrm{d} x \mathrm{~d} y \\
& \quad=\iint_{\Omega} \delta u_{i} t_{i} \mathrm{~d} x \mathrm{~d} y
\end{aligned}
$$

where the geometric strain-displacement relations are those in Eq. (7) and the following mixed constitutive law is used:

$$
\begin{aligned}
& {\left[\begin{array}{l}
\sigma_{\alpha \beta}^{(C)} \\
\epsilon_{i 3}^{(C)}
\end{array}\right]^{(p)}=\left[\begin{array}{ll}
C_{\alpha \beta \gamma \delta} & C_{\alpha \beta j 3} \\
C_{i 3 \alpha \beta} & C_{i 3 j 3}
\end{array}\right]^{(p)}\left[\begin{array}{l}
\epsilon_{\gamma \delta}^{(G)} \\
\sigma_{j 3}
\end{array}\right]} \\
& \text { with }\left\{\begin{array}{l}
C_{\alpha \beta \gamma \delta}=\widetilde{C}_{\alpha \beta \gamma \delta}-\widetilde{C}_{\alpha \beta i 3} \widetilde{C}_{i 3 j 3}^{-1} \widetilde{C}_{j 3 \gamma \delta} \\
C_{\alpha \beta i 3}=\widetilde{C}_{\alpha \beta j 3} \widetilde{C}_{j 3 i 3}^{-1} \\
C_{i 3 \alpha \beta}=-\widetilde{C}_{i 3 j 3}^{-1} \widetilde{C}_{j 3 \alpha \beta} \\
C_{i 3 j 3}=\widetilde{C}_{i 3 j 3}^{-1}
\end{array}\right.
\end{aligned}
$$

With reference to the vector notation of Eq. (9), the constitutive relation Eq. (12) is written as

$$
\left[\begin{array}{l}
\sigma_{\omega}^{(C)} \\
\epsilon_{n}^{(C)}
\end{array}\right]^{(p)}=\left[\begin{array}{ll}
C_{\omega \omega} & C_{\omega n} \\
-C_{\omega n}^{\top} & C_{n n}
\end{array}\right]^{(p)}\left[\begin{array}{l}
\epsilon_{\omega}^{(G)} \\
\sigma_{n}
\end{array}\right]
$$

and the internal virtual work expressed by RMVT Eq. (11) reads

$$
\begin{aligned}
\delta W_{i n t}= & \iint_{\Omega}\left\{\int_{H} \delta \epsilon_{\omega}^{(G) \top} \sigma_{\omega}^{(C)}+\delta \epsilon_{n}^{(G) \top} \sigma_{n}+\delta \sigma_{n}^{\top}\left(\epsilon_{n}^{(G)}-\epsilon_{n}^{(C)}\right) \mathrm{d} z\right\} \mathrm{d} x \mathrm{~d} y \\
= & \iint_{\Omega}\left\{\int_{H} \delta \epsilon_{\omega}^{(G) \top} C_{\omega \omega}^{(p)} \epsilon_{\omega}+\delta \epsilon_{\omega}^{(G) \top} C_{\omega n}^{(p)} \sigma_{n}+\delta \epsilon_{n}^{(G) \top} \sigma_{n}+\delta \sigma_{n}^{\top} \epsilon_{n}^{(G)}\right. \\
& \left.+\delta \sigma_{n}^{\top} C_{\omega n}^{(p) \top} \epsilon_{\omega}^{(G)}-\delta \sigma_{n}^{\top} C_{n n}^{(p)} \sigma_{n} \mathrm{~d} z\right\} \mathrm{d} x \mathrm{~d} y
\end{aligned}
$$

\subsection{GUF approximations in the sublaminate}

Let $\mathcal{U}$ designate the generic variable for which approximations are introduced, i.e., $\mathcal{U} \in\left\{u_{i}\right\}$ for the displacement-based approach of the PVD and $\mathcal{U} \in\left\{u_{i}, \sigma_{i 3}\right\}$ for the mixed RMVT approach. If all $N_{p}^{k}$ plies constituting the $k$ th sublaminate are described in ESL manner, the approximations are expressed in terms of the sublaminate $z_{k}$ coordinate by means of the following compact notation introduced by Demasi [22]:

$\mathcal{U}^{k}\left(x, y, z_{k}\right)=\sum_{\alpha_{\mathcal{U}}=0}^{N_{\mathcal{U}}^{k}} F_{\alpha_{\mathcal{U}}}\left(z_{k}\right) \hat{\mathcal{U}}_{\alpha_{\mathcal{U}}}^{k}(x, y)$

If the $N_{p}^{k}$ plies of the sublaminate are described in a LW manner, the ply-specific coordinate $z_{p}$ is used:

$\mathcal{U}^{p}\left(x, y, z_{p}\right)=\sum_{\alpha_{\mathcal{U}}=0}^{N_{\mathcal{U}}^{k}} F_{\alpha_{\mathcal{U}}}\left(z_{p}\right) \hat{\mathcal{U}}_{\alpha_{\mathcal{U}}}^{p}(x, y)$

and the resulting through-thickness approximation for the $k$ th sublaminate is obtained from the assembly of all ply contributions:

$\mathcal{U}^{k}\left(x, y, z_{k}\right)=\sum_{p=p_{1}^{k}}^{p_{N}^{k}} \mathcal{U}^{p}\left(x, y, z_{p}\right)=\sum_{p=p_{1}^{k}}^{p_{N}^{k}}\left\{\sum_{\alpha_{\mathcal{U}}=0}^{N_{\mathcal{U}}^{k}} F_{\alpha_{\mathcal{U}}}\left(z_{p}\right) \hat{\mathcal{U}}_{\alpha_{\mathcal{U}}}^{p}(x, y)\right\}$

where $p_{1}^{k}$ and $p_{N}^{k}$ are the indices of the first (bottom) and last (top) ply of the $k$ th sublaminate, respectively. The key point of S-GUF is that the order of the expansion $N_{\mathcal{U}}^{k}$ depends not only on the variable (subscript $\mathcal{U}$ ) as in GUF, but also on the sublaminate $k$. This allows to express different approximations for different sublaminates. Note also that the same order $N_{\mathcal{U}}^{k}$ is used for all $N_{p}^{k}$ plies within the same $k$ th sublaminate. It is finally emphasized that the generic variable $\mathcal{U}^{k}$ may be described within all plies of the $k$ th sublaminate in an 
ESL or a LW manner, and that this choice can be made for each sublaminate independently.

\subsubsection{The employed approximating functions}

The approximating functions $F_{\alpha_{u}}$ are expressed in terms of a non-dimensional coordinate $\zeta \in[-1,1]$ that will be specified depending on the ESL or LW description employed inside the sublaminate: $\zeta=\zeta_{k}$ for an ESL description and $\zeta=\zeta_{p}$ for a LW description. The employed functions are defined as follows:

$$
\begin{aligned}
\text { if } N_{\mathcal{U}}^{k}=0: \quad F_{0}(\zeta)=1 \\
\text { if } N_{\mathcal{U}}^{k} \geqslant 1: \quad F_{0}(\zeta)=\frac{1+\zeta}{2} ; \quad F_{1}(\zeta)=\frac{1-\zeta}{2} ; \\
\quad F_{r}(\zeta)=P_{r}(\zeta)-P_{r-2}(\zeta) \quad \text { for } r=2,3, \ldots N_{\mathcal{U}}^{k}
\end{aligned}
$$

where $P_{r}(\zeta)$ is Legendre's polynomial of order $r$ that can be defined recursively from following relations $[54]$

$P_{0}(\zeta)=1 ; \quad P_{1}(\zeta)=\zeta ; P_{r+1}(\zeta)=\frac{(2 r+1) \zeta P_{r}(\zeta)-r P_{r-1}(\zeta)}{r+1}$

The polynomial functions in Eq. (17) are used in the proposed sublaminate version of GUF for representing both LW and ESL approximations inside the sublaminate $k$. This is different with respect to the original CUF and GUF, in which Taylor's polynomial expansion is used for ESL descriptions, see, e.g., [55]. Fig. 3 shows the resulting functions $F_{\alpha}(\zeta)$ for an expansion order $N=4$. The choice of the polynomial functions Eq. (17) is based on convenience only. Note that Eq. (17) defines the linear Lagrange polynomials $F_{0}(\zeta)=F_{t}(\zeta)$ and $F_{1}(\zeta)=F_{b}(\zeta)$ that interpolate the values $\mathcal{U}_{0}=\mathcal{U}_{t}=\mathcal{U}(\zeta=1)$ at the top and $\mathcal{U}_{1}=\mathcal{U}_{b}=\mathcal{U}(\zeta=-1)$ at the bottom of the interval. The chosen functions combine thus the feature of Lagrange's linear interpolation, which allows a direct access at interface values, and the orthogonality of higher-order Legendre's polynomials.

\subsubsection{Zig-Zag function for ESL models}

If an ESL description is used for the whole laminate $\left(N_{l}=1, z_{k}=z\right)$, Murakami's Zig-Zag Function (MZZF) $F_{Z Z}\left(\zeta_{p}\right)=(-1)^{p} \zeta_{p}$ can be superimposed to the approximation described by Eq. (17) in order to introduce a slope discontinuity at each interface between plies. The approximation in Eq. (14) is thus enhanced as follows:

$u_{i}(x, y, z)=\sum_{\alpha_{u_{i}}=0}^{N_{u_{i}}^{k}} F_{\alpha_{u_{i}}}(z) \hat{u}_{i \alpha_{u_{i}}}(x, y)+F_{Z Z}\left(\zeta_{p}\right) \hat{u}_{i z Z}(x, y)$

Note that MZZF can be here used independently for the three displacement components, see also [35].

\subsection{Construction of plate model arrays}

According to the variable kinematics approach realized by Unified Formulations, the arrays for the plate model are obtained as an assembly of sublaminate arrays, which are in turn obtained as an

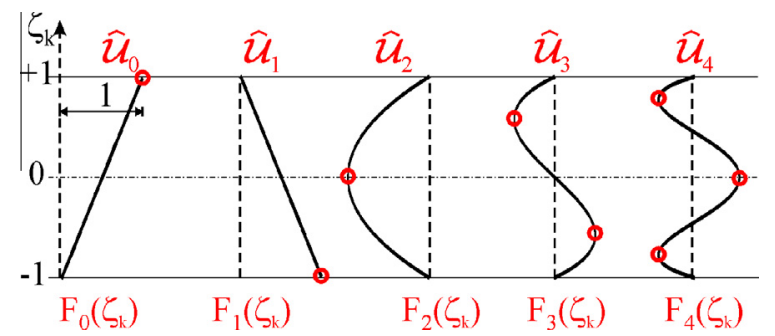

Fig. 3. Functions $F_{\alpha}\left(\zeta_{k}\right)(\alpha \in\{0,4\})$ used for the polynomial approximation in the $k$ th layer. assembly of elementary building blocks (kernels) related to the assumptions introduced at ply level.

\subsubsection{Generic kernel of the ply}

The gradient relations Eq. (7) defining the strains in terms of the displacements are substituted in the variational statement Eq. (6) or Eq. (11). Now let $\mathcal{U}_{s}$ be a generic unknown function and $\delta \mathcal{U}_{q}$ the virtual variation of a generic unknown function, i.e., $\mathcal{U}_{(q, s)} \in\left\{u_{i}\right\}$ for the PVD Eq. (6) and $\mathcal{U}_{(q, s)} \in\left\{u_{i}, \sigma_{i 3}\right\}$ for the RMVT Eq. (11).

The generic contribution of $\delta \mathcal{U}_{q}$ and $\mathcal{U}_{s}$ to the virtual internal work defined by Eq. (10b) or Eq. (13b) is expressed in terms of ply contributions in order to cope with the material-dependent coefficient $c_{Q S}^{(p)}$ :

$\delta W_{\text {int }}\left(\delta \mathcal{U}_{q}, \mathcal{U}_{s}, c_{Q S}^{(p)}\right)=\sum_{k=1}^{N_{l}}\left\{\sum_{p=p_{1}^{k}}^{p_{N}^{k}} \delta W_{i n t}^{p}\left(\delta \mathcal{U}_{q}^{p}, \mathcal{U}_{s}^{p}, c_{Q S}^{(p)}\right)\right\}$

Upon introducing the S-GUF approximation Eq. (14) or Eq. (15), this generic contribution of the ply is expressed as

$$
\begin{aligned}
\delta W_{\text {int }}^{p}\left(\delta \mathcal{U}_{q}^{p}, \mathcal{U}_{s}^{p}, c_{Q S}^{(p)}\right)= & \iint_{\Omega}\left(\partial_{\alpha}^{(q)} \delta \hat{\mathcal{U}}_{q \alpha_{q}}^{p}(x, y)\right) Z_{\mathcal{U}_{q} \mathcal{U}_{s} Q S}^{p \alpha_{\mathcal{u}} \beta u_{s}} \\
& \times\left(\partial_{\alpha}^{(s)} \hat{\mathcal{U}}_{s \beta_{q}}^{p}(x, y)\right) \mathrm{d} x \mathrm{~d} y
\end{aligned}
$$

with the integral over the ply thickness defined as

$Z_{\mathcal{U}_{q} \mathcal{u}_{s} Q S}^{p \alpha_{u_{q}(z)} \beta u_{\mathcal{S}^{(, z)}}}=c_{Q S}^{(p)} \int_{z_{p}^{b}}^{z_{p}^{t}}\left[\partial_{z}^{(q)} F_{\alpha_{q}} \partial_{z}^{(s)} F_{\beta_{s}}\right] \mathrm{d} z$

The appropriate definition of the thickness function has to be accounted for in the computation of the Z-integrals of the sublaminate, i.e., $F=F\left(z_{k}\right)$ for an ESL description and $F=F\left(z_{p}\right)$ for a LW description, as in Eqs. (14) and (15).

The generic contribution possesses different particular instantiations, whose explicit expressions depend on the variational statement, the nature of the variables $\mathcal{U}_{q}$ and $\mathcal{U}_{s}$ and the coefficient $c_{Q S}^{(p)}$. For the PVD case, $c_{Q S}^{(p)}=\widetilde{C}_{Q S}^{(p)}$ is always a material stiffness, with $(Q, S) \in\{1,6\}^{2}$ according to Eq. (10a). For the RMVT case, however, displacement and stress variables may be directly workconjugated without the need for a material parameter; therefore, $c_{Q S}^{(p)}$ may be a material parameter $C_{Q S}^{(p)}$ according to Eq. (13a), or a unitary value. Furthermore, some variables may be differentiated with respect to the in-plane directions $x_{\alpha}$ or the transverse direction $x_{3}=z$; this is indicated in Eq. (21) by the symbols $\partial_{\alpha}^{(q, s)}$ and $\partial_{z}^{(q, s)}$, respectively, where the superscript specifies the concerned variable.

The notation for the integrals over the ply thickness $Z_{\mathcal{U}_{q} \mathcal{U}_{s} Q S}^{p \alpha_{\mathcal{u}_{s}} \beta_{\mathcal{U}_{s}}}$ is the same as in GUF [22,23]. A tilde is used to discern the integrals $\widetilde{Z}$ related to the PVD formulation and issued from the stiffness coefficients $\widetilde{C}_{Q S}$. These $1 \times 1$ terms are expanded to form the contribution of the ply to the virtual internal work term Eq. (21a) upon cycling over the indices $\alpha_{\mathcal{U}_{q}}=0,1, \ldots N_{q}^{k}$ and $\beta_{\mathcal{U}_{s}}=0,1, \ldots N_{s}^{k}$. Detailed expressions for all particular instantiations of the generic contribution (21) related to the PVD and RMVT frameworks can be found in $[22,23]$, respectively, along with the expansion procedure to form the ply contribution.

\subsubsection{Assembly at sublaminate level: ESL and LW descriptions}

The procedure for assembling the ply-contributions of the $Z$ integrals to form the sublaminate contributions depends on the ESL or LW description of the concerned variables inside the sublaminate. The same standard procedure of the established CUF 


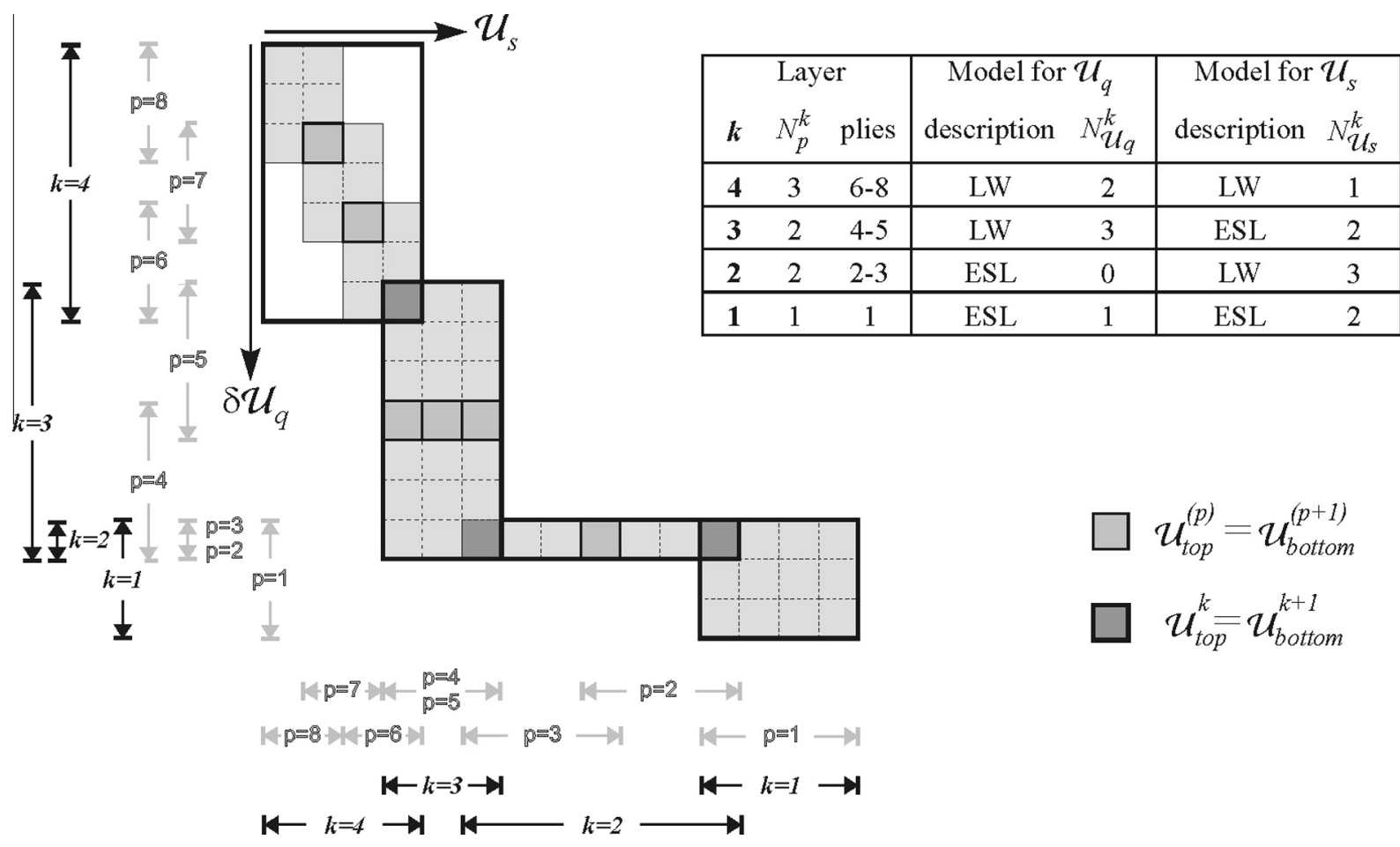

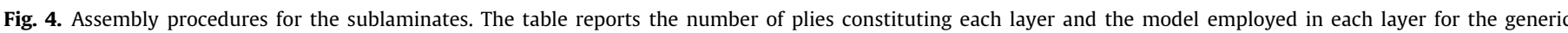
variables $\mathcal{U}_{q}$ and $\mathcal{U}_{s}$.

Table 1

Some ESL models and their designation and number of DOF within the present S-GUF.

\begin{tabular}{|c|c|c|c|}
\hline Model & $\begin{array}{l}\text { S-GUF } \\
\text { acronym }\end{array}$ & Remarks & DOF \\
\hline CLT & $\mathrm{ED}_{1,0}$ & $\begin{array}{l}\text { No transv. shear flexibility: } \\
1 / G_{\alpha 3}=0\end{array}$ & 5 \\
\hline FSDT & $\mathrm{ED}_{1,0}$ & $\begin{array}{l}\text { Shear correction factor } \kappa=5 / 6 \\
\text { is used }\end{array}$ & 5 \\
\hline RMZC [41] & $\mathrm{M}_{\mathrm{E} 1 Z, 0}^{\mathrm{L} 2,}$ & $\begin{array}{l}u_{\alpha} \mathrm{ESL}+\mathrm{MZZF}, \sigma_{\alpha 3} \mathrm{LW}, \sigma_{33}=0 ; \\
\mathrm{SC}^{\dagger}\end{array}$ & 7 \\
\hline TSDT [30] & $\mathrm{EM}_{3,0}^{2,}$ & $u_{i} \mathrm{ESL}, \sigma_{\alpha 3} \mathrm{ESL}, \sigma_{33}=0 ; \mathrm{SC}^{\dagger}$ & 9 \\
\hline LCW [33] & $\mathrm{ED}_{3,2}$ & 3D constitutive law & 11 \\
\hline ZZE PVD 889 [35] & $\mathrm{ED}_{8 \mathrm{Z}, 9}$ & $u_{\alpha} \mathrm{ESL}+\mathrm{MZZF}, u_{3} \mathrm{ESL}$ & 30 \\
\hline $\mathrm{EMC}_{221}^{332}[55]$ & $\mathrm{M}_{\mathrm{E} 2, \mathrm{E} 1}^{\mathrm{L} 3 \mathrm{~L} 2}$ & $u_{i} \mathrm{ESL}, \sigma_{i 3} \mathrm{LW}, \mathrm{SC}^{\dagger}$ & 8 \\
\hline
\end{tabular}

$\dagger$ SC indicates Static Condensation of the transverse stress unknowns.

and GUF is used and details can be found, e.g., in [55]. The chosen interpolation functions Eq. (17) allow to impose the continuity condition in a straight-forward manner for both ESL and LW assembling processes.

\subsubsection{Assembly of sublaminates at laminate level}

The sublaminate contributions of different layers are always assembled in a LW sense with the standard procedure that enforces the continuity of the generic variable $\mathcal{U}^{k}$ at the interfaces between adjacent layers, see, e.g., [54]. Note that the employed interpolation functions Eq. (17) permit to follow the same standard assembly procedure even if an ESL description is used inside the sublaminates. Since the novelty of the proposed S-GUF is that different approximations may be chosen for different sublaminates, an example of a resulting assembly scheme for 4 sublaminates, each consisting of an arbitrary number of plies and employing very different approximations, is illustrated in Fig. 4. By indicating with $N_{\mathcal{U}_{(q, s)}}^{k}$ the number of DOF for the generic variables $\mathcal{U}_{(q, s)}$ in the sublaminate $k$, the resulting array for the $Z$-integral for the whole multilayer that describes the virtual internal work contribution Eq. (21a) has the size $\left(\left(\sum_{k=1}^{N_{l}} N_{\mathcal{U}_{q}}^{k}\right)-\left(N_{l}-1\right)\right) \times$ $\left(\left(\sum_{k=1}^{N_{l}} N_{\mathcal{U}_{s}}^{k}\right)-\left(N_{l}-1\right)\right)$.

\subsection{Governing equations of the $2 \mathrm{D}$ model: Navier-type solution}

Once the model along the thickness coordinate $z$ has been introduced, the unknown functions of the remaining 2D problems are $\hat{\mathcal{U}}_{\mu_{\mathcal{U}}}(x, y)$, where the index $\mu_{\mathcal{U}}$ ranges over all terms that define the expansion across the whole multilayered plate, i.e., $\mu_{\mathcal{U}} \in\left\{1,\left(\sum_{k=1}^{N_{l}} N_{\mathcal{U}}^{k}\right)-\left(N_{l}-1\right)\right\}$. In this work the Navier-type solution method is adopted, which permits to find exact solutions to problems involving simply-supported, rectangular and specially orthotropic plates [11]. The strong form of the 2D governing equations are first obtained from the integral over the in-plane domain $\Omega$ in Eqs. (6) and (11) by integrating by parts the terms whose virtual variations are differentiated with respect to the in-plane coordinates $x_{\alpha}$. An exact solution of the resulting set of PDE and of the associated boundary conditions is then given - under the above mentioned restricting circumstances - by the following harmonic solution:

$$
\begin{aligned}
& \left\{\hat{u}_{1 \mu_{u_{1}}}, \hat{\sigma}_{5 \mu_{\sigma_{5}}}\right\}(x, y)=\left\{U_{1 \mu_{u_{1}}}, T_{5 \mu_{\sigma_{5}}}\right\} \cos \left(\frac{m \pi x}{a}\right) \sin \left(\frac{n \pi y}{b}\right) \\
& \left\{\hat{u}_{2 \mu_{u_{2}}}, \hat{\sigma}_{4 \mu_{\sigma_{4}}}\right\}(x, y)=\left\{U_{2 \mu_{u_{2}}}, T_{4 \mu_{\sigma_{4}}}\right\} \sin \left(\frac{m \pi x}{a}\right) \cos \left(\frac{n \pi y}{b}\right) \\
& \left\{\hat{u}_{3 \mu_{u_{3}}}, \hat{\sigma}_{3 \mu_{\sigma_{3}}}\right\}(x, y)=\left\{U_{3 \mu_{u_{3}}}, T_{3 \mu_{\sigma_{3}}}\right\} \sin \left(\frac{m \pi x}{a}\right) \sin \left(\frac{n \pi y}{b}\right)
\end{aligned}
$$

with $x \in[0, a]$ and $y \in[0, b]$. Substitution of the above Navier-type solution into the set of PDE yields finally the algebraic system whose unknowns are the parameters of the thickness approximation. The resulting matrix system for the PVD case has the following form 


$$
\left[\begin{array}{lll}
\mathbf{K}_{U_{1} U_{1}} & \mathbf{K}_{U_{1} U_{2}} & \mathbf{K}_{U_{1} U_{3}} \\
& \mathbf{K}_{U_{2} U_{2}} & \mathbf{K}_{U_{2} U_{3}} \\
\operatorname{sym} & & \mathbf{K}_{U_{3} U_{3}}
\end{array}\right]\left[\begin{array}{l}
\mathbf{U}_{1} \\
\mathbf{U}_{2} \\
\mathbf{U}_{3}
\end{array}\right]=\left[\begin{array}{l}
\mathbf{R}_{1} \\
\mathbf{R}_{2} \\
\mathbf{R}_{3}
\end{array}\right]
$$

while the form of that obtained from the RMVT case is
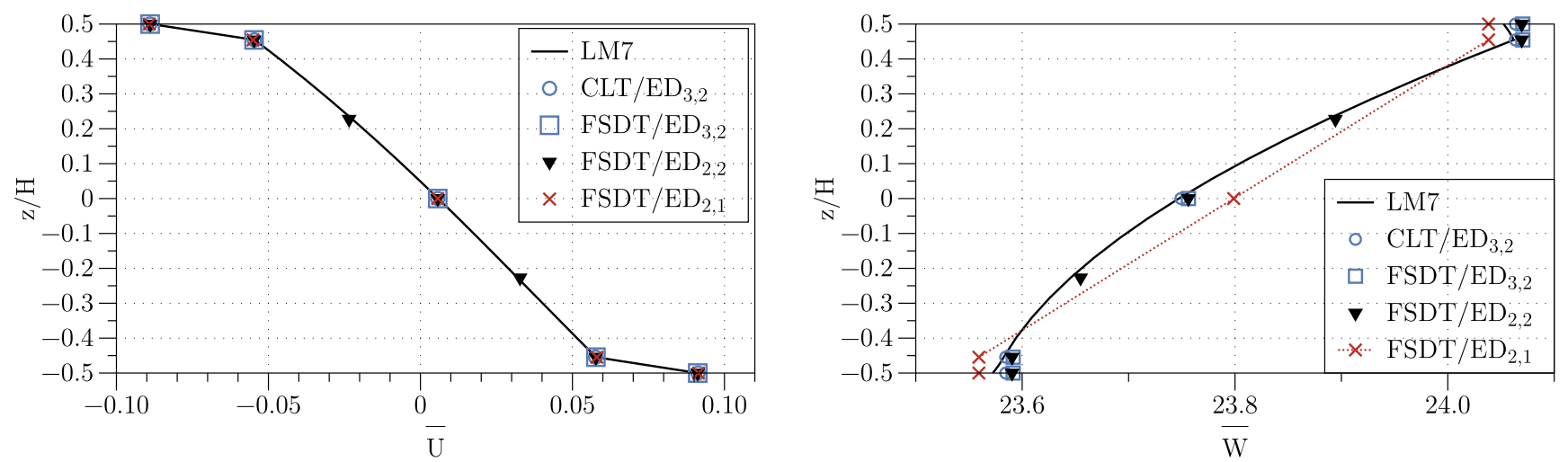

(a) Displacements $\bar{U}(z), \bar{W}(z)$
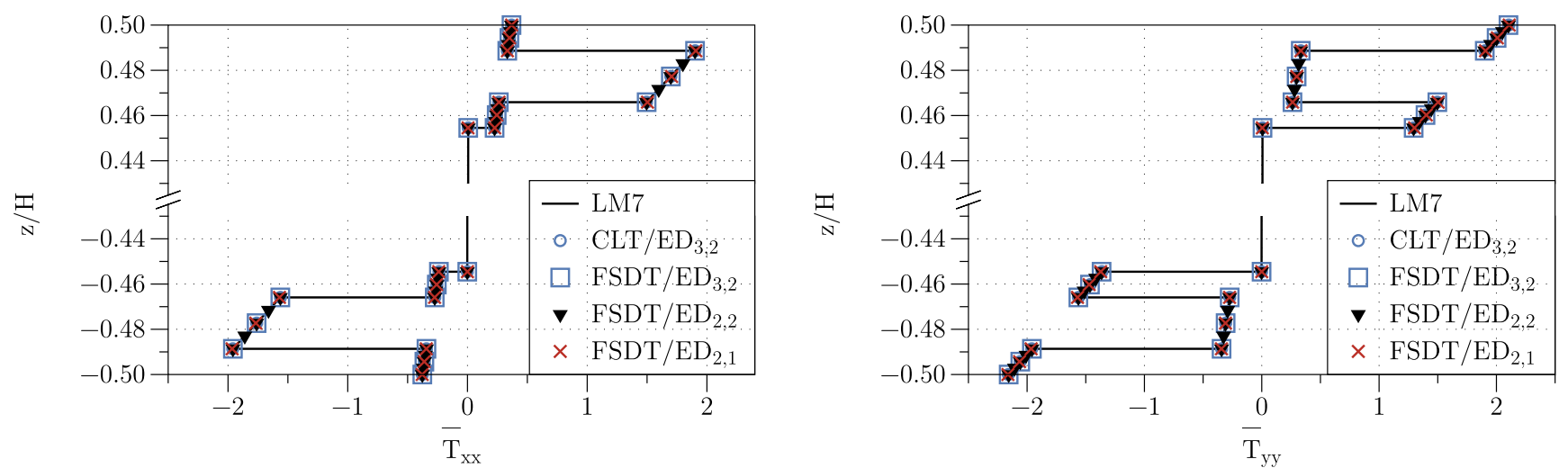

(b) In-plane stresses $\bar{T}_{x x}(z), \bar{T}_{y y}(z)$ (zoom on laminated faces)
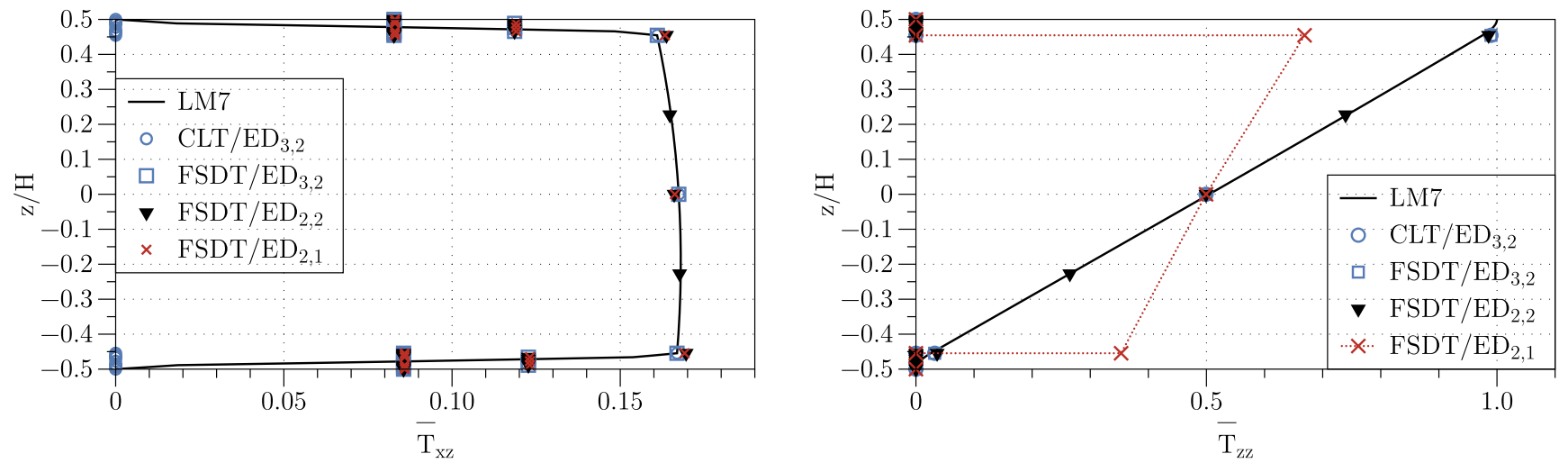

(c) Transverse stresses $\bar{T}_{x z}(z), \bar{T}_{z z}(z)$

Fig. 5. BM1: assessment of PVD-based 3-layers models. 


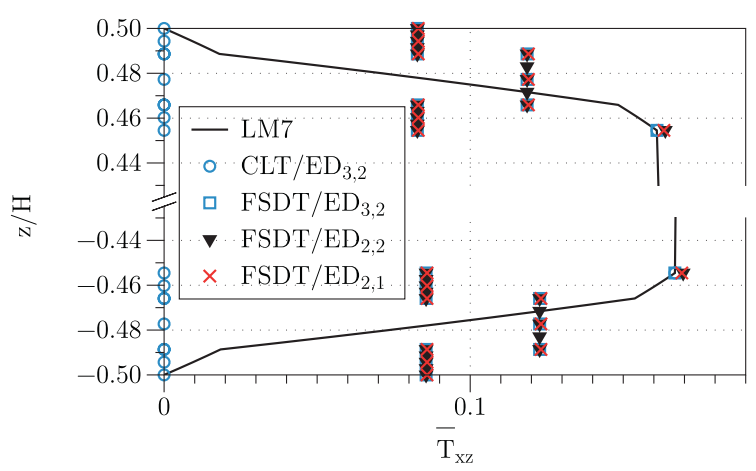

Fig. 6. BM1: detail of the transverse shear stress in the laminated faces.

which can be recast in the following partitioned form

$$
\left[\begin{array}{ll}
\mathbf{K}_{U U} & \mathbf{K}_{U T} \\
\mathbf{K}_{T U} & \mathbf{K}_{T T}
\end{array}\right]\left[\begin{array}{l}
\mathbf{U} \\
\mathbf{T}
\end{array}\right]=\left[\begin{array}{l}
\mathbf{R} \\
\mathbf{0}
\end{array}\right]
$$

Stress unknowns $T$ can be statically condensed out, which yields the following reduced matrix system

$$
\tilde{\mathbf{K}}_{U U} \mathbf{U}=\mathbf{R} \quad \text { with } \quad \tilde{\mathbf{K}}_{U U}=\mathbf{K}_{U U}-\mathbf{K}_{U T}\left(\mathbf{K}_{T T}\right)^{-1} \mathbf{K}_{T U}
$$

Within the proposed formulation, the static condensation can be carried out for separate sublaminates, or for the whole multilayered plate [56]. Note that in the framework of the employed Navier-type solution, the static condensation carried out at multilayered level introduces no approximation. Once the static condensation is carried out, the modified stiffness matrix $\tilde{\mathbf{K}}_{U U}$ incorporates in an integral sense the approximations formulated for the transverse stress field, e.g., including the transverse stress continuity at ply interfaces or the satisfaction of zero transverse shear stress at top and bottom.

\section{Naming convention}

A dedicated nomenclature is introduced for designating the various plate models that can be formulated by means of the proposed Sublaminate-GUF. For each sublaminate, a notation similar to that proposed by Demasi is adopted as follows:

- Capital D and capital M stay for PVD and RMVT models, respectively.

- The order of the polynomial expansion of the displacements and the stresses are given in the subscript and superscript, respectively. For the sake of simplicity, the same order $N_{u_{1}}^{k}=N_{u_{2}}^{k}$ will be employed for both in-plane displacement components $u_{\alpha}^{k}$; analogously we set $N_{\sigma_{13}}^{k}=N_{\sigma_{23}}^{k}$ for both transverse shear components $\sigma_{\alpha 3}^{k}$. Only two indexes are hence necessary for denoting the polynomial expansion order: $N_{u_{\alpha}}^{k}, N_{u_{3}}^{k}$ in the subscript and $N_{\sigma_{\alpha 3}}^{k}, N_{\sigma_{33}}^{k}$ in the superscript. In addition to the standard expansion orders of CUF and GUF, the present S-GUF implements the constant distribution, which corresponds to a 0th order $\left(N_{\mathcal{U}}=0\right)$, and offers the possibility of discarding a transverse stress component, which is indicated by a dot. So, plane stress models formulated within RMVT have $N_{\sigma_{33}}=$.

- As in standard CUF and GUF notation, capital $\mathrm{E}$ and L indicate ESL and LW descriptions, respectively. However, the capital E or $\mathrm{L}$ are reported in the sub- or superscript just before the order of expansion used for the displacement and stress variables. In fact, differently from standard CUF and GUF, the present S-GUF allows an ESL description for the transverse stress variables as well. If all displacement and stress variables are described in the same way, the capital $\mathrm{E}$ or $\mathrm{L}$ is reported in the model name just before the $\mathrm{D}$ or the $\mathrm{M}$.
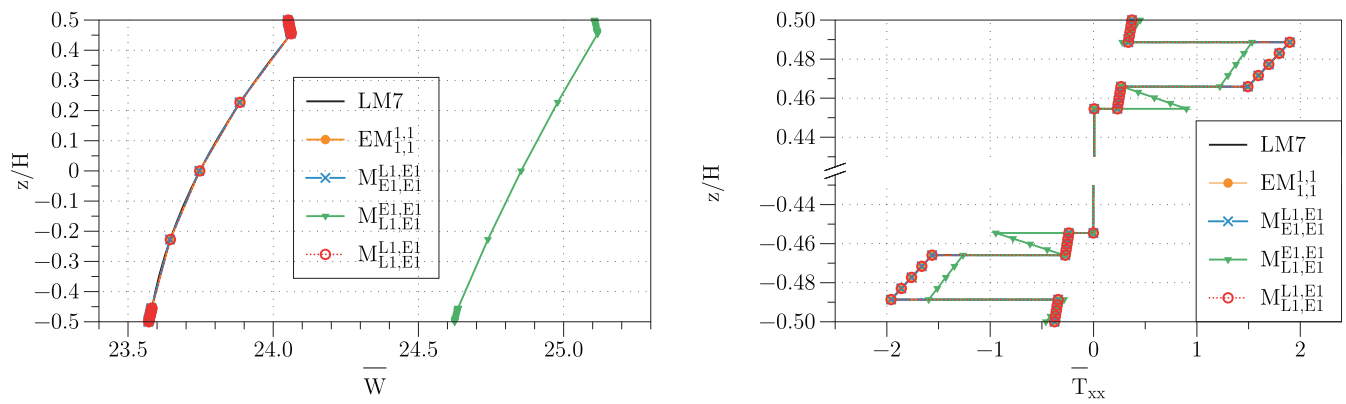

(a) Bending response $\bar{W}(z)$ and $\bar{T}_{x x}(z)$ (detail of laminated faces)
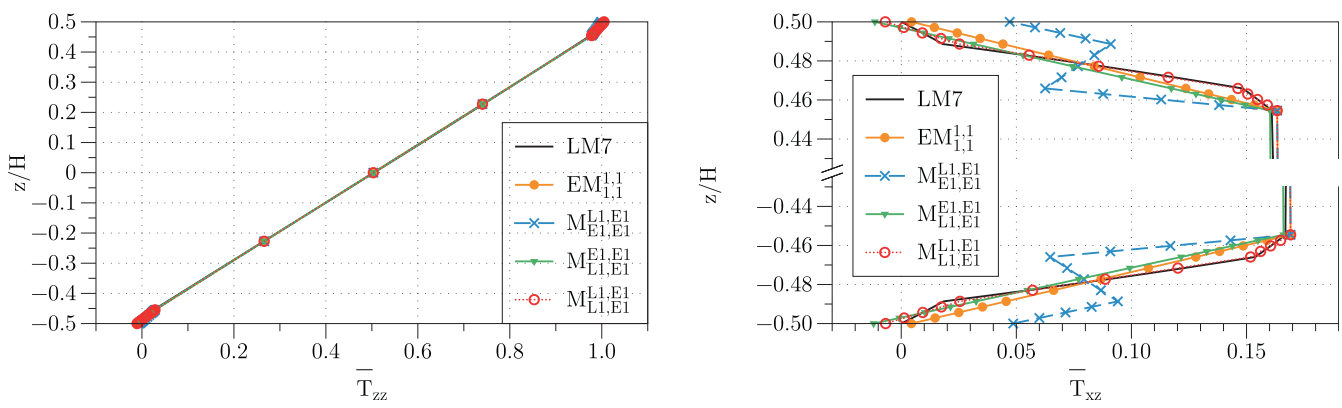

(b) Transverse stresses $\bar{T}_{z z}(z)$ and $\bar{T}_{x z}(z)$ (detail of laminated faces)

Fig. 7. BM1: assessment of several linear RMVT models for the faces (core model: $\mathrm{EM}_{2,2}^{1,1}$ ). 
- A similar convention is used for the capital $\mathrm{Z}$ indicating the inclusion of MZZF: the capital $\mathrm{Z}$ is reported in the subscript after the expansion order of the concerned displacement component, and it appears in the model name just after the D or the M only if MZZF is used for all displacement components.

Many models proposed in literature can be represented within the proposed S-GUF. Table 1 reports some known ESL models and their representation within the present S-GUF. Only one sublaminate is used for representing ESL models. It should be noted that the number of DOF reported in Table 1 corresponds to that of the present S-GUF implementation. For instance, CLT has the same DOF number as FSDT since the vanishing transverse shear strain is enforced by penalyzing the transverse shear stiffness. Moreover, the DOF number reported in Table 1 for RMVT-based models is that of the displacement-based model obtained through the Static Condensation (SC) of the transverse stress unknowns.

When the plate is composed of several sublaminates, the acronyms are reported from the top sublaminate to the bottom one, separated by a slash. Three-layers models for sandwich plates are thus defined by specifying the model employed for the faces and the core. In the numerical evaluations discussed in the following section, the same model will be used for both top and bottom faces even if the present S-GUF is evidently not limited to this classical choice.

\section{Numerical results}

The main features of the proposed S-GUF will be highlighted by two benchmark problems that address the bending response of simply-supported rectangular and orthotropic plates. The first benchmark illustrates the versatility of the proposed S-GUF as a tool for obtaining hierarchic accuracies along with a computational cost reduction compared to classical Unified Formulations. In the second benchmark, S-GUF is used to reproduce different models available in literature for consistently assess them against the challenging problem proposed by Meyer-Piening.

\subsection{BM1: sandwich plate with laminated faces}

The following results shall demonstrate the capability of the proposed S-GUF to reduce the computational cost while still preserving the hierarchic model accuracies offered by the established CUF and GUF. For this, a new benchmark problem is considered that involves a representative sandwich plate with laminated composite faces:

Geometry: Core thickness $h_{c}=20$ [mm], thickness of bottom and top faces $h_{f}=1$ [mm] (face-to-core thickness ratio $\left.h_{f} / h_{c}=0.05\right)$. The plate is square of dimension $a \times a$ with aspect ratio $a / H=10$.

Loading: Bi-sinusoidal pressure load $p(x, y)=p_{0} \sin \left(\frac{\pi x}{a}\right) \sin \left(\frac{\pi y}{b}\right)$ at top surface.

Materials: Each face consists of four CFRP plies with stacking sequence $[90,0]_{s}$ and following properties: $h_{p}=0.25[\mathrm{~mm}]$; $E_{L}=107[\mathrm{GPa}], E_{T}=15[\mathrm{GPa}], G_{L T}=4.3[\mathrm{GPa}], G_{T T}=3[\mathrm{GPa}]$, $v_{L T}=0.3, v_{T T}=0.49$. An isotropic foam core is considered with $E_{c}=105[\mathrm{MPa}], G_{c}=40[\mathrm{MPa}]$ (therefore, $v_{c}=0.3125$ is taken). The face-to-core stiffness ratio is hence FCSR $=\frac{E_{f x}}{E_{c}} \approx 585$.

Results: The following non-dimensional results are considered: in-plane displacement $\bar{U}(z)=u_{1}\left(x=0, y=\frac{b}{2}, z\right) \frac{E_{T}}{p_{0} H S^{3}}$, lateral displacement $\bar{W}(z)=u_{3}\left(x=\frac{a}{2}, y=\frac{b}{2}, z\right) \frac{100 E_{T}}{p_{0} H S^{4}}, \quad$ in-plane stresses $\left\{\bar{T}_{x x}(z), \bar{T}_{y y}(z)\right\}=\left\{\sigma_{x x}\left(x=\frac{a}{2}, y=\frac{b}{2}, z\right), \sigma_{y y}\left(x=\frac{a}{2}, y=\frac{b}{2}, z\right)\right\} \frac{1}{p_{0} S^{2}}$, transverse shear stress $\bar{T}_{x z}(z)=\sigma_{x z}\left(x=0, y=\frac{b}{2}, z\right) \frac{1}{p_{0} S}$, transverse normal stress $\bar{T}_{z z}(z)=\sigma_{z z}\left(x=\frac{a}{2}, y=\frac{b}{2}, z\right) \frac{1}{p_{0}}$.

The hierarchic accuracy of S-GUF models is first applied in the PVD framework to identify the most opportune model for the core. So, several displacement-based, three-layers models are assessed in Fig. 5 with respect to the LM7 model, a high-order mixed model that is capable of accurately recovering a full 3D response, see also [57]. The laminated faces are modeled as CLT or FSDT, whereas several different refined kinematics including transverse stretch are used for the core. A very good agreement of the in-plane response (displacement $\bar{U}$, bending stresses $\bar{T}_{x x}$ and $\bar{T}_{y y}$ ) is noticed among all models. The out-of-plane response $\bar{W}$ and $\bar{T}_{z z}$ inside the core appears accurate as long as the linear term of the transverse stretch is retained, i.e., $N_{u_{3}} \geqslant 2$ should be chosen for the core layer. The core model with $N_{u_{\alpha}}=N_{u_{3}}=2$, which includes a linear transverse shear and a linear transverse stretch, provides the best accuracy-to-computational cost ratio. As far as the local transverse stress response inside the laminated face is concerned, FSDT can only provide an estimate of their mean value across the whole face, see Fig. 6.

A model assessment for the laminated faces is next proposed by referring to RMVT-based models. RMVT is here addressed in order to explore the possibility of recovering accurate transverse stress results in the laminated faces with low-order polynomial expansions. According to the previous analysis, the RMVT model for the core is selected to be $\mathrm{EM}_{2,2}^{1,1}$. All considered face models include the transverse normal stress. In a first approximation, a linear ESL approximation is used for the transverse deflection $u_{3}$ and stress $\sigma_{33}$. Fig. 7 illustrates the results obtained with linear approximations and different ESL/LW descriptions of the in-plane displacements $u_{\alpha}$ and transverse shear stresses $\sigma_{\alpha 3}$. From the results reported in Fig. 7 the following conclusions can be drawn concerning the choice of the model for the laminated faces:

- the use of a linear ESL model for the transverse normal stress and transverse normal displacement is adequate;

- if $u_{\alpha}$ is described ESL and $\sigma_{\alpha 3}$ is LW, the bending response is accurate but the local transverse shear stresses distribution is inaccurate;

- if $u_{\alpha}$ is described LW and $\sigma_{\alpha 3}$ is ESL, the bending response is inaccurate even if the local transverse shear stress distribution is meaningful;

- a full ESL model provides rather accurate results for both the global bending and the local transverse shear stress;

- accuracy is enhanced using a LW description for both $u_{\alpha}$ and $\sigma_{\alpha 3}$.

It is stressed that these considerations hold for the considered case study involving thin, laminated faces and that extrapolation to other configurations deserves preliminary investigation. For instance, it is known that thick faces call for quadratic transverse shear stress distributions.

In order to point out the computational cost saving that can be achieved with the proposed S-GUF, some models considered for

\section{Table 2}

Models assessed in BM1 and respective DOF number. Values in parentheses indicate the DOF number after Static Condensation (SC) of transverse stress unknowns.

\begin{tabular}{|c|c|c|c|c|c|}
\hline \multicolumn{2}{|l|}{ S-GUF } & \multicolumn{2}{|l|}{ GUF } & \multicolumn{2}{|l|}{ CUF } \\
\hline Faces/core & $\mathrm{DOF}(\mathrm{SC})$ & Model & $\mathrm{DOF}(\mathrm{SC})$ & Model & $\mathrm{DOF}(\mathrm{SC})$ \\
\hline FSDT/ED ${ }_{2,2}$ & 13 & $\mathrm{LD}_{2,2}$ & 45 & LD2 & 45 \\
\hline $\mathrm{EM}_{1,1}^{1,1} / \mathrm{EM}_{2,2}^{1,1}$ & 27 (15) & $\mathrm{LM}_{2}^{1,1}$ & 69 (45) & LM2 & $90(45)$ \\
\hline $\mathrm{M}_{\mathrm{L} 1, \mathrm{E} 1}^{\mathrm{L} 1, \mathrm{E} 1} / \mathrm{EM}_{2,2}^{1,1}$ & 43 (23) & & & & \\
\hline
\end{tabular}


this benchmark problem are reported in Table 2 along with their DOF number. The DOF number in parenthesis corresponds to that of the system once the transverse stress unknowns are statically condensed out. PVD and RMVT-based S-GUF models consist of 3 sublaminates, one for each laminated face and one for the core. Equivalent CUF and GUF models are considered by retaining a full LW description with the highest expansion orders used in the SGUF. Note that classical CUF and GUF do not offer the possibility of an ESL description of the transverse stresses.

Fig. 8 compares the global deflection and the local transverse shear stress in the laminated faces, obtained by the S-GUF

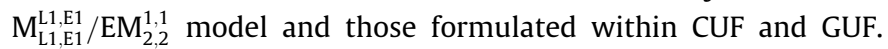
Note that the GUF $\mathrm{LD}_{2,2}$ model exactly coincide with the CUF LD2 model. The values in Table 2 in combination with the results in Fig. 8 show clearly that S-GUF models can be conveniently used for reducing the number of DOF without substantially affecting the accuracy. It should be finally noted that RMVT-based models require special attention in the determination of the number of stress parameters compared to the number of displacement DOFs [58]: GUF models with $N_{u_{\alpha}}=N_{u_{3}}>N_{\sigma_{\alpha 3}}=N_{\sigma_{33}}$, such as the considered $\mathrm{LM}_{2,2}^{1,1}$, are known to provide a spuriously oscillating displacement field, which for this reason is omitted from Fig. 8(a). S-GUF provides also in this context more freedom for selecting the appropriate model.

\subsection{BM2: Meyer-Piening benchmark}

A more discriminant case study for sandwich plate models has been provided by Meyer-Piening [59] and involves an unsymmetric rectangular sandwich plate subjected to a localized pressure load:

Geometry: Rectangular plate with $a=100$ [mm], $b=200$ [mm] and thickness $H=12$ [mm]. The sandwich is unsymmetric with the bottom face thickness $h_{1}=0.5[\mathrm{~mm}]$, top face thickness $h_{3}=0.1[\mathrm{~mm}]$ and core thickness $h_{c}=11.4[\mathrm{~mm}]$.

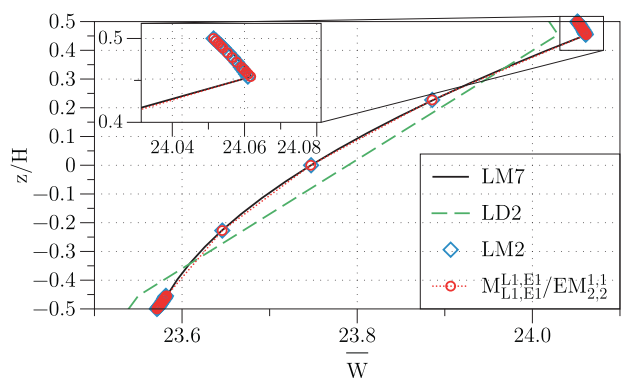

(a) Bending response $\bar{W}(z)$

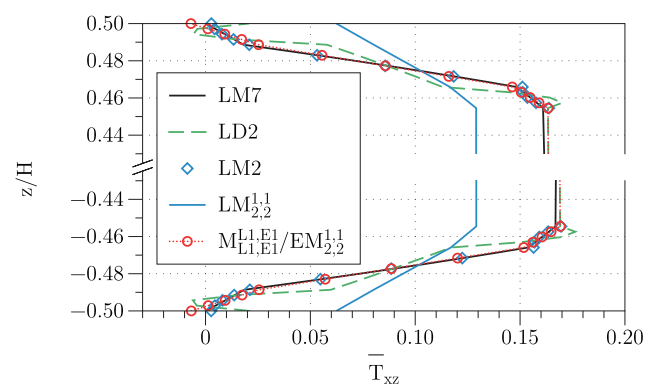

(b) Transverse shear stress $\bar{T}_{x z}(z)$

Fig. 8. BM1, comparison of an S-GUF model with equivalent classical GUF and CUF models.

Table 3

BM2: comparison of present LM7 results with the 3D solution reported by Meyer-Piening [59].

\begin{tabular}{|c|c|c|c|c|c|c|}
\hline & $z$ & $u_{z}[\mathrm{~mm}]$ & $\sigma_{x x}[\mathrm{MPa}]$ & $\sigma_{y y}[\mathrm{MPa}]$ & $\sigma_{x y}[\mathrm{MPa}]$ & $\sigma_{z z}^{(\dagger)}[\mathrm{MPa}]$ \\
\hline 3D [59] & $\begin{array}{l}6 \\
5.9^{+} \\
-5.5^{-} \\
-6\end{array}$ & $\begin{array}{l}-3.78 \\
- \\
- \\
-2.14\end{array}$ & $\begin{array}{l}-624 \\
580 \\
-138 \\
146\end{array}$ & $\begin{array}{l}-380 \\
353 \\
-91 \\
97\end{array}$ & $\begin{array}{l}128 \\
-126 \\
7 \\
-8\end{array}$ & $\begin{array}{l}-1.1 \\
-0.85 \\
-0.18 \\
0\end{array}$ \\
\hline LM7 & $\begin{array}{l}6 \\
5.9^{+} \\
-5.5^{-} \\
-6\end{array}$ & $\begin{array}{l}-3.78 \\
-3.78 \\
-2.14 \\
-2.14\end{array}$ & $\begin{array}{l}-624 \\
580 \\
-138 \\
146\end{array}$ & $\begin{array}{l}-371 \\
344 \\
-93 \\
98\end{array}$ & $\begin{array}{l}129 \\
-127 \\
6 \\
-6\end{array}$ & $\begin{array}{l}-1.1 \\
-0.85 \\
-0.18 \\
0\end{array}$ \\
\hline
\end{tabular}

(†) Values referred to Fourier series expansion with 31 terms.

Table 4

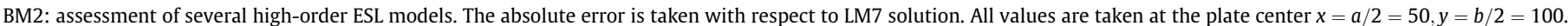

\begin{tabular}{|c|c|c|c|c|c|c|c|}
\hline & $z$ & $u_{z}[\mathrm{~mm}]$ & (\% error) & $\sigma_{x x}[\mathrm{MPa}]$ & (\% error) & $\sigma_{y y}[\mathrm{MPa}]$ & (\% error) \\
\hline \multirow[t]{2}{*}{ RMZC } & 6 & -0.03 & (99) & -28 & $(96)$ & -21 & (91) \\
\hline & -6 & -0.03 & (99) & 7 & (95) & 5 & (96) \\
\hline \multirow[t]{2}{*}{ TSDT } & 6 & -3.36 & (11) & -37 & (94) & -27 & (89) \\
\hline & -6 & -3.36 & (57) & 44 & (70) & 33 & (74) \\
\hline \multirow[t]{2}{*}{ LCW } & 6 & -0.45 & $(88)$ & -52 & (92) & -49 & $(80)$ \\
\hline & -6 & -0.44 & (79) & 124 & (15) & 85 & (33) \\
\hline \multirow[t]{2}{*}{ ED9 } & 6 & -3.23 & (15) & -513 & (18) & -192 & (20) \\
\hline & -6 & -1.84 & (14) & 112 & (23) & 95 & $(25)$ \\
\hline \multirow[t]{2}{*}{$\mathrm{ED}_{8 \mathrm{Z}, 9}$} & 6 & -3.45 & (9) & -547 & (12) & -200 & (17) \\
\hline & -6 & -2.02 & (6) & 132 & (10) & 109 & (14) \\
\hline \multirow[t]{2}{*}{ EDZ8 } & 6 & -3.60 & (4) & -610 & (2) & -235 & (2) \\
\hline & -6 & -2.01 & (6) & 138 & (5) & 116 & (9) \\
\hline \multirow[t]{2}{*}{ LM7 } & 6 & -3.78 & - & -624 & - & -241 & - \\
\hline & -6 & -2.14 & - & 146 & - & 127 & - \\
\hline
\end{tabular}




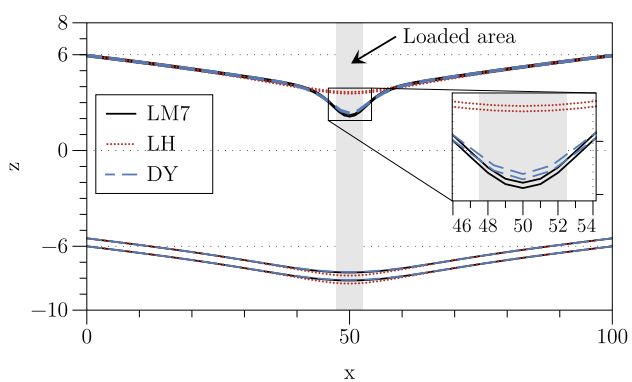

(a) Central plane cut along $x$-axis at $y=100$

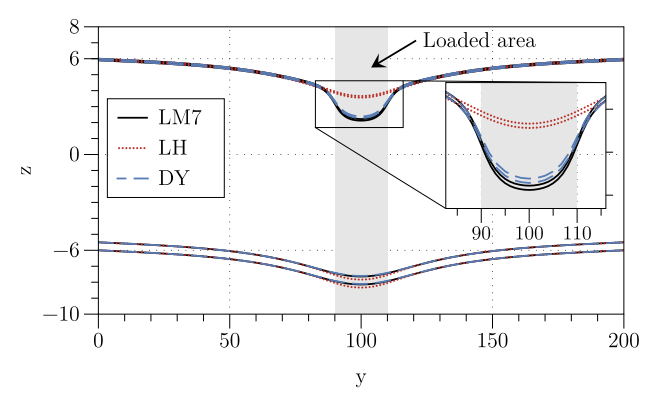

(b) Central plane cut along $y$-axis at $x=50$

Fig. 9. Displaced configuration of Meyer-Piening sandwich under local pressure load.

Table 5

Three-layered models for sandwich panels and their representation within S-GUF assessed in the Meyer-Piening benchmark BM2.

\begin{tabular}{lll}
\hline Model & S-GUF (faces/core) & DOF \\
\hline LH [45] & FSDT/FSDT & 9 \\
HL [51] & CLT/ED & 12 \\
DY [53] & FSDT/ED & 12 \\
PFK [52] & $\mathrm{CLT} / \mathrm{ED}_{3,2}$ & 12 \\
PP [46] & $\mathrm{ED}_{3,2} / \mathrm{ED}_{3,2}$ & 15 \\
\hline
\end{tabular}

Loading: Localized uniform pressure $p_{0}=1[\mathrm{MPa}]$ acting at the top surface on an area of $5 \times 20\left[\mathrm{~mm}^{2}\right]$ centered at $(x, y)=\left(\frac{a}{2}, \frac{b}{2}\right)$. Materials: Faces with $E_{f x}=70[\mathrm{GPa}], \quad E_{f y}=71$ [GPa], $E_{f z}=69[\mathrm{GPa}], \quad G_{f}=26[\mathrm{GPa}]$ and $v_{f}=0.3$; core with $E_{c x}=E_{c y}=3[\mathrm{MPa}], E_{c z}=2.8[\mathrm{MPa}], G_{c}=1[\mathrm{MPa}]$ and $v_{c}=0.25$. Results: Dimensioned results (displacements in [mm], stresses in [MPa]) are taken at selected points and along selected paths.

The high face-to-core stiffness ratio $\left(\frac{E_{f}}{E_{c}} \approx 10^{4}\right)$ and the localized load make this case study particularly challenging for sandwich plate models. This benchmark is next used to assess several models established in the literature and reproduced by the proposed SGUF.

Present solutions are obtained as Fourier series expansion with 101 terms in both $x$ and $y$ directions, as suggested by MeyerPiening [59]. Table 3 shows that the results obtained by the high-order LM7 model - transverse deflection $w(x=50, y=100)$, in-plane stresses $\sigma_{x x}(50,100), \sigma_{y y}(50,92.5)$ and $\sigma_{x y}(47.5,90)$, and transverse normal stress $\sigma_{z z}(50,100)$ - successfully compare against those extracted from [59]. The values are reported for the plate top and bottom surfaces $(z= \pm 6)$ as well as for both face/core interfaces, i.e., at $z=5.9^{+}$for the top interface and at $z=-5.5^{-}$for the bottom one (stresses are evaluated in the faces). Note that the values for $\sigma_{z z}$ refer to a Fourier series expansion with only 31 terms, see also [59].

Table 4 proposes an assessment of several high-order ESL models (see also Table 1 ) for the bending response with respect to the LM7 solution. Transverse displacement $u_{z}$ and in-plane direct stresses $\sigma_{x x}$ and $\sigma_{y y}$ are reported at the plate center $(x=50, y=100)$ and at its top $(z=6)$ and bottom $(z=-6)$ surfaces. Despite the Zig-Zag function, the enhanced first-order model

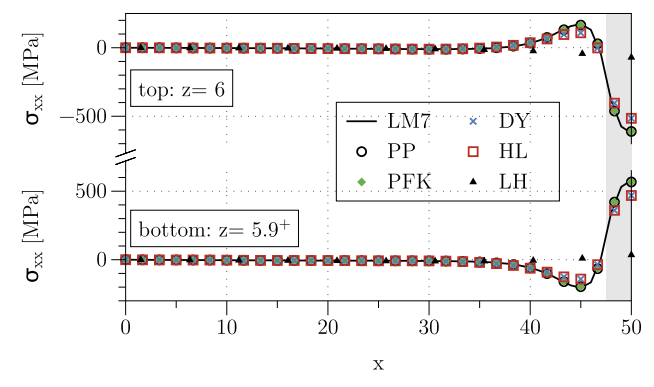

(a) $\sigma_{x x}(x, y=100)$ in top face

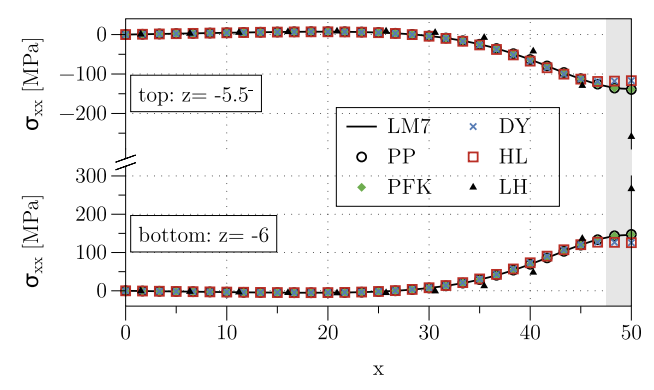

(c) $\sigma_{x x}(x, y=100)$ in bottom face

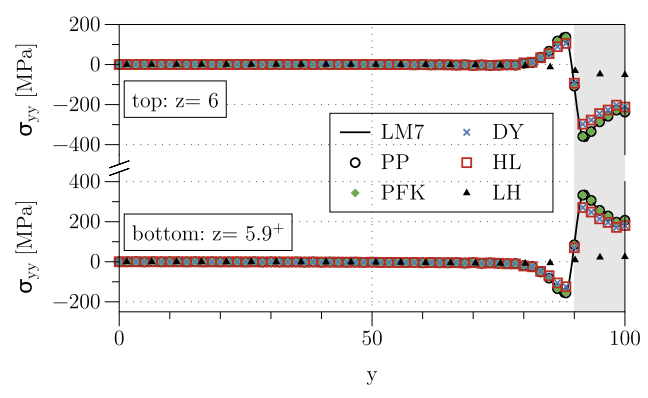

(b) $\sigma_{y y}(x=50, y)$ in top face

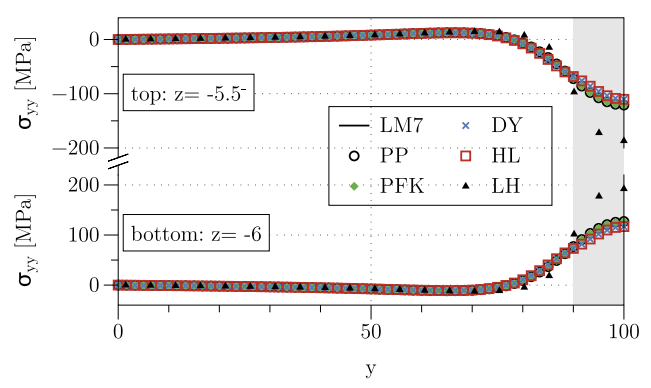

(d) $\sigma_{y y}(x=50, y)$ in bottom face

Fig. 10. BM2: Bending stresses at bounding surfaces of top and bottom sandwich faces. 


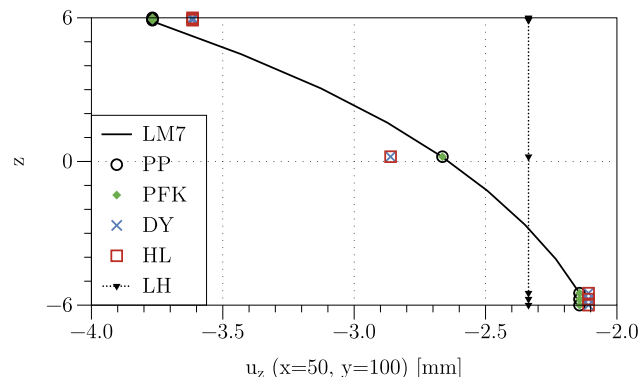

(a) Deflection $u_{z}$

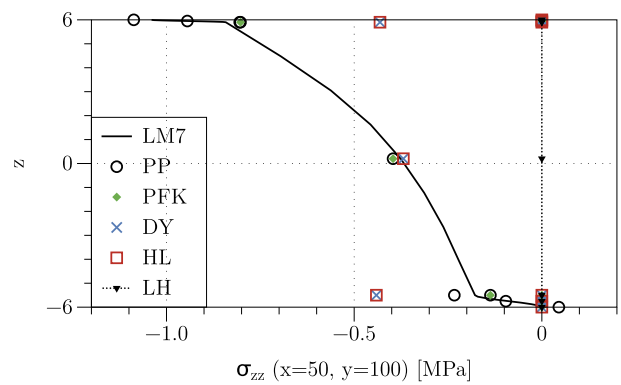

(c) Transverse normal stress $\sigma_{z z}$

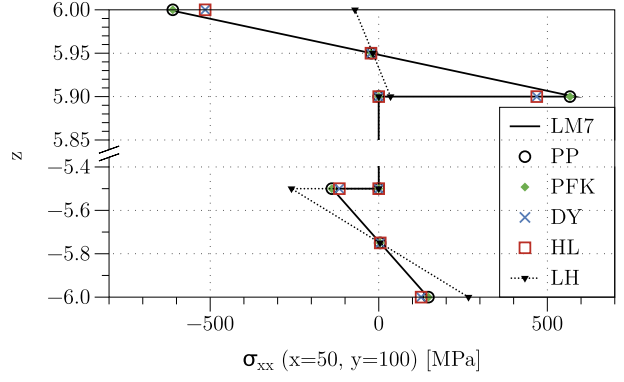

(b) Bending stress $\sigma_{x x}$ (detail of faces)

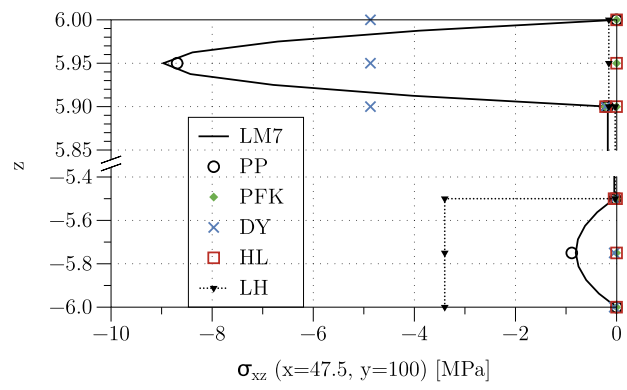

(d) Transverse shear stress $\sigma_{x z}$ (detail of faces)

Fig. 11. BM2: Through-thickness response at plate center $(x=50, y=100)(\mathrm{a})-(\mathrm{c})$ and at edge of loaded region $(x=47.5, y=100)(\mathrm{d})$.

RMZC is seen to completely fail in this severe problem because of the low-order polynomial expansion and the plane stress assumption. Third-order models such as TSDT and LCW behave generally better than RMZC, but still provide unacceptable results with errors up to $90 \%$. One may notice that TSDT provides a more accurate deflection than LCW, whereas LCW is slightly more accurate in the prediction of the bending stresses compared to TSDT. Three high-order displacement-based models involving the same DOF number (30) are finally compared. The increasing accuracy of $\mathrm{ED} 9, \mathrm{ED}_{8 \mathrm{Z}, 9}$ and EDZ8 highlights that the use of MZZF for all displacement components, including the transverse deflection, is of great benefit.

Finally, an assessment is proposed of some displacement-based three-layers plate models that have been introduced in the literature review. The considered models are summarized in Table 5 along with their S-GUF representation and associated DOF number.

Fig. 9 reports the deformed shape of the sandwich plate in sections cut in the central planes $y=100$ and $x=50$. The indentation of the top face caused by the locally applied pressure load is clearly visible. One notices that the LH model fails to reproduce this local deformation due to the FSDT model adopted in the core, which neglects the transverse normal deformation. Fig. 10 reports the evolutions of the bending stresses $\sigma_{x x}$ (Fig. 10 left) and $\sigma_{y y}$ (Fig. 10 right) along the $x$ and $y$ coordinate, respectively. The stresses are taken at the top and bottom surfaces of the top and bottom face of the sandwich. The localized bending at the top face is well represented by all models that include the transverse normal stretch inside the core. These models can distinguish the different gradients that characterize the response at the top face and at the bottom face. It can be further seen that the HL and DY models have some difficulties in following the steep gradients occurring at the edge of the loaded area: higher order kinematics, such as the third-order one used by the PFK and PP models, is indeed required in presence of rapid load variations.

Through-thickness distributions are reported in Fig. 11. The inplane coordinates $(x, y)$ are considered where the response has its maximum magnitude. So, lateral deflection $u_{z}$, bending stress $\sigma_{x x}$ and transverse normal stress $\sigma_{z z}$ are taken in the plate center $(x=50, y=100)$, see Fig. 11(a)-(c), while the transverse shear stress $\sigma_{x z}$ is taken at the edge of the loaded region $(x=47.5, y=100)$, see Fig. 11(d). All considered three-layers models provide reasonable estimates for the lateral deflection and bending stress. The transverse stress response is more challenging. The Meyer-Piening benchmark involves a parabolic transverse shear stress inside the faces, with a maximum peak of approximately $2 \%$ of the maximum bending stress, see Fig. 11(d). In order to grasp this stress, FSDT and CLT are not sufficient and HSDT should be used in the faces. The transverse normal stress is of even smaller magnitude $\left(\max \left(\sigma_{z z}\right) \approx 0.1 \sigma_{x z}\right)$. However, as already shown, it is necessary to include the transverse stress effects at least inside the core, in order to correctly estimate the rapid gradients of the response due to the localized load.

\section{Conclusions}

This paper has presented an extension of the Generalized Unified Formulation (GUF) in which different approximations can be introduced in different groups of plies (sublaminates) constituting the multilayered plate. This Sublaminate-GUF (S-GUF) enhances the modeling flexibility of classical GUF and appears particularly useful for sandwich panels because the very different material and geometric properties call for dedicated assumptions for the faces and the core. S-GUF permits to substantially reduce the computational cost within the hierarchic model definition that characterizes the usefulness of Unified Formulations. Furthermore, several sandwich plate models available in the literature can be represented within one single computer program and thus consistently assessed against each other. Further work already in preparation addresses the study of viscoelastic sandwich plates, the inclusion of interface slip as well as of piezoelectric coupling. The extension to curved panel geometries shall not pose any substantial issues. 


\section{References}

[1] Kapania RK, Raciti S. Recent advances in analysis of laminated beams and plates. Part 1: Shear effects and buckling. Part 2: vibrations and wave propagation. AIAA J 1989;27(923-934):935-46.

[2] Noor AK, Burton WS. Assessment of computational models for multilayered composite shells. Appl Mech Rev 1990;43:67-97.

[3] Reddy JN, Robbins Jr DH. Theories and computational models for composite laminates. Appl Mech Rev 1994;47:147-69.

[4] Noor AK, Burton WS. Computational models for sandwich panels and shells. Appl Mech Rev 1996;49:155-99.

[5] Carrera E. Theories and finite elements for multilayered, anisotropic composite plates and shells. Arch Comput Methods Eng 2002;9:87-140.

[6] Sayyad AS, Ghugal YM. On the free vibration analysis of laminated composite and sandwich plates: A review of recent literature with some numerical results. Compos Struct 2015;129:177-201.

[7] Reddy JN. An evaluation of equivalent-single-layer and layerwise theories of composite laminates. Compos Struct 1993;25:21-35.

[8] Carrera E. Historical review of zig-zag theories for multilayered plates and shells. Appl Mech Rev 2003;56:287-308.

[9] Allen HG. Analysis and design of structural sandwich panels. Pergamon Press; 1969.

[10] Reddy JN. A generalization of two-dimensional theories of laminated composite laminates. Comm Appl Numer Methods 1987:3:173-80.

[11] Reddy JN. Mechanics of laminated composite plates and shells: theory and analysis. 2nd Edition. CRC Press; 2004.

[12] Carrera E. Theories and finite elements for multilayered plates and shells: a unified compact formulation with numerical assessment and benchmarking. Arch Comput Methods Eng 2003;10:215-96.

[13] Carrera E, Cinefra M, Petrolo M, Zappino E. Finite element analysis of structures through unified formulation. John Wiley \& Sons Ltd; 2014.

[14] Carrera E. An assessment of mixed and classical theories on global and local response of multilayered orthotropic plates. Compos Struct 2000;50:183-98.

[15] Demasi L, Yu W. Assess the accuracy of the variational asymptotic plate and shell analysis using the generalized unified formulation. Mech Adv Mater Struct 2013;20:227-41.

[16] D'Ottavio M, Vidal P, Valot E, Polit O. Assessment of plate theories for free-edge effects. Compos B 2013;48:111-21.

[17] Carrera E, Cinefra M, Lamberti A, Petrolo M. Results on best theories for metallic and laminated shells including Layer-Wise models. Compos Struct 2015;126:285-98

[18] Vidal P, Gallimard L, Polit O. Assessment of a composite beam finite element based on the proper generalized decomposition. Compos Struct 2012;94:1900-10.

[19] Biscani F, Giunta G, Belouettar S, Carrera E, Hu H. Variable kinematic plate elements coupled via Arlequin method. Int J Numer Methods Eng 2012;91:1264-90.

[20] Wenzel C, Vidal P, D’Ottavio M, Polit O. Coupling of heterogeneous kinematics and finite element approximations applied to composite beam structures. Compos Struct 2014;116:177-92.

[21] Reissner E. On a certain mixed variational theorem and a proposed application. Int J Numer Methods Eng 1984:20:1366-8.

[22] Demasi L. $\infty^{3}$ hierarchy plate theories for thick and thin composite plates: The generalized unified formulation. Compos Struct 2008;84:256-70.

[23] Demasi L. $\infty^{6}$ Mixed plate theories based on the generalized unified formulation. Part I: governing equations. Compos Struct 2009;87:1-11.

[24] Demasi L. Partially Layer Wise advanced Zig Zag and HSDT models based on the Generalized Unified Formulation. Eng Struct 2013;53:63-91.

[25] Botshekanan Dehkordi M, Cinefra M, Khalili SMR, Carrera E. Mixed LW/ESL models for the analysis of sandwich plates with composite faces. Compos Struct 2013;98:330-9.

[26] Messina A. Analyses of freely vibrating cross-ply laminated plates in conjunction with adaptive global piecewise-smooth functions (A-GPSFs). Int J Mech Sci 2015;90:179-89.

[27] Bhaskar K, Varadan TK. The contradicting assumptions of zero transverse normal stress and strain in the thin plate theory: a justification. J Appl Mech 2001;68:660-2.

[28] Birman V, Bert CW. On the choice of shear correction factor in sandwich structures. J Sandwich Struct Mater 2002;4:83-95.

[29] Ambartsumyan SA. Theory of anisotropic plates. Technomic 1970.
[30] Reddy JN. A simple higher-order theory for laminated composite plates. J Appl Mech 1984:51:745-52.

[31] Touratier M. A refined theory for laminated shallow shells. Int J Solids Struct 1992;29:1401-15.

[32] Hildebrand FB, Reissner E, Thomas GB, Notes on the foundations of the theory of small displacements of orthotropic shells, Tech. Rep. 1833, National Advisory Committee for Aeronautics; 1949.

[33] Lo KH, Christensen RM, Wu EM. A high-order theory of plate deformation Part 1: Homogeneous plates - Part 2: laminated plates. J Appl Mech 1977;44 (663-668):669-76.

[34] Carrera E, Brischetto S. Analysis of thickness locking in classical, refined and mixed multilayered plate theories. Compos Struct 2008;82:549-62.

[35] Demasi L. Partially Zig-Zag advanced higher order shear deformation theories based on the Generalized Unified Formulation. Compos Struct 2012;94:363-75

[36] Pandit MK, Sheikh AH, Singh BN. An improved higher order zigzag theory for the static analysis of laminated sandwich plate with soft core. Finite Elem Anal Des 2008;44:602-10.

[37] Tessler A, Di Sciuva M, Gherlone M. A refined zigzag beam theory for composite and sandwich beams. J Compos Mater 2009;43:1051-81.

[38] Vidal P, Polit O, D'Ottavio M, Valot E. Assessment of the refined sinus plate finite element: Free edge effect and Meyer-Piening sandwich test. Finite Elem Anal Des 2014;92:60-71.

[39] Murakami H. Laminated composite plate theory with improved in-plane response. J Appl Mech 1986;53:661-6.

[40] Toledano A, Murakami H. A high-order laminated plate theory with improved in-plane response. Int J Solids Struct 1987;23:111-31.

[41] Carrera E. $C^{0}$ Reissner-Mindlin multilayered plate elements including zig-zag and interlaminar stress continuity. Int $J$ Numer Methods Eng 1996;39:1797-820.

[42] Carrera E. On the use of Murakami's zig-zag function in the modeling of layered plates and shells. Comput Struct 2004;82:541-54.

[43] Brischetto S, Carrera E, Demasi L. Improved bending analysis of sandwich plates using a zig-zag function. Compos Struct 2009;89:408-15.

[44] Carrera E, Brischetto S. A survey with numerical assessment of classical and refined theories for the analysis of sandwich plates. Appl Mech Rev 2009;62:1-17.

[45] Librescu L, Hause T. Recent developments in the modeling and behavior of advanced sandwich constructions: a survey. Compos Struct 2000;48:1-17.

[46] Pai PF, Palazotto AN. A higher-order sandwich plate theory accounting for 3-D stresses. Int J Solids Struct 2001;38:5045-62.

[47] D'Ottavio M, Polit O. Linearized global and local buckling analysis of sandwich struts with a refined quasi-3D model. Acta Mech 2015;226:81-101.

[48] Reissner E. On small bending and stretching of sandwich-type shells. Int J Solids Struct 1977;13:1293-300.

[49] Kanematsu HH, Hirano Y, Ijama H. Bending and vibration of CFRP-faced rectangular sandwich plates. Compos Struct 1988;10:145-63.

[50] Frostig Y, Baruch M. High-order buckling analysis of sandwich beams with transversely flexible core. J Eng Mech 1993;119:476-95.

[51] Hohe J, Librescu L. Advances in the structural modeling of elastic sandwich panels. Mech Adv Mater Struct 2004;11:395-424.

[52] Phan CN, Frostig Y, Kardomateas GA. Analysis of sandwich beams with a compliant core and with in-plane rigidity - Extended high-order sandwich panel theory versus elasticity. J Appl Mech 2012;79.

[53] Dawe DJ, Yuan WX. Overall and local buckling of sandwich plates with laminated faceplates, part I: analysis. Comput Methods Appl Mech Eng 2001;190:5197-213.

[54] Demasi L. $\infty^{6}$ Mixed plate theories based on the generalized unified formulation. Part II: layerwise theories. Compos Struct 2009;87:12-22.

[55] Demasi L. $\infty^{6}$ Mixed plate theories based on the generalized unified formulation. Part III: advanced mixed high order shear deformation theories. Compos Struct 2009;87:183-94.

[56] Carrera E. Developments, ideas and evaluations based upon Reissner's Mixed Variational Theorem in the modeling of multilayered plates and shells. Appl Mech Rev 2001:54:301-29.

[57] Demasi L. 2D, Quasi 3D and 3D exact solutions for bending of thick and thin sandwich plates. J Sandwich Struct Mater 2008;10:271-310.

[58] Demasi L. $\infty^{6}$ Mixed plate theories based on the generalized unified formulation. Part V: results. Compos Struct 2009;88:1-16.

[59] Meyer-Piening H-R. Application of the elasticity solution to linear sandwich beam, plate and shell analysis. J Sandwich Struct Mater 2004:6:295-312. 